\title{
Polyhedral Analysis for Concentrator Location Problems
}

\author{
MARTINE LABBÉ \\ mlabbe@ulb.ac.be \\ Université Libre de Bruxelles, Département d'Informatique, Boulevard du Triomphe CP 210/01, 1050 Bruxelles, \\ Belgium
}

HANDE YAMAN

hyaman@bilkent.edu.tr

Bilkent University, Department of Industrial Engineering, Bilkent 06800 Ankara, Turkey

Received August 26, 2004; Revised May 4, 2005; Accepted July 11, 2005

Published online: 23 March 2006

\begin{abstract}
The concentrator location problem is to choose a subset of a given terminal set to install concentrators and to assign each remaining terminal node to a concentrator to minimize the cost of installation and assignment. The concentrators may have capacity constraints. We study the polyhedral properties of concentrator location problems with different capacity structures. We develop a branch and cut algorithm and present computational results.
\end{abstract}

Keywords: concentrator location, polyhedral analysis, branch and cut

\section{Introduction}

In this paper, we study the polytopes of the concentrator location problems with different capacity restrictions. The general problem is the Quadratic Capacitated Concentrator Location Problem (QCL). It is defined as follows. We are given a set of terminal nodes and a traffic matrix whose entries represent the amount of traffic between pairs of terminals. This traffic is routed by concentrators (also called switches, multiplexers and routers). We choose a subset of the terminals to be the concentrator locations. Each node that receives a concentrator is assigned to itself and each one of the remaining nodes is assigned to exactly one concentrator node. The aim of the problem is to minimize the cost of locating concentrators and the cost of assigning terminals to concentrators.

The concentrators have capacities. The sum of the fixed demands of nodes that are assigned to a concentrator and the traffic between nodes that are assigned to this concentrator and the nodes that are assigned to other concentrators cannot exceed the capacity of the concentrator. Often in telecommunication applications, the fixed demand of a node is the sum of the traffic with this node as origin or destination. Then the capacity of a concentrator is in terms of the traffic on the links adjacent to that concentrator (see [7, 15]).

We also study two special cases of the QCL. If all terminal nodes can be assigned to a single concentrator, the problem is called the Uncapacitated Concentrator Location 
Problem (UCL). The second case arises when the capacity constraints concern only the sum of the fixed demands of nodes that are assigned to the same concentrator. We call this problem the Linear Capacitated Concentrator Location Problem (LCL).

The aim of this paper is to study the polyhedral properties of the concentrator location problems. These polyhedral results are then used to develop a branch and cut algorithm to solve QCL. It is interesting to note that the polytopes of concentrator location problems are closely related to the polyhedra of location problems with routing cost (see [15]). More precisely, the facet defining inequalities of the concentrator location polytopes define also facets of the polyhedra of location problems with routing cost.

Closely related are the facility location problems. For a survey on these problems and their variants which have applications in telecommunications, see Gourdin et al. [11].

Aardal et al. [1] and Leung and Magnanti [16] study the polytope of the capacitated facility location problem. A branch and cut algorithm based on the results of [1] is given in [2]. Deng and Simchi-Levi [9] consider the same problem with the additional constraint that each customer can be served by a single facility. Some of the results of this paper are reviewed in Section 4.

This paper is organized as follows. In Section 2, we give a formal definition of the QCL, derive a formulation and discuss its variants based on the capacity restrictions. In Section 3 , we present linearizations for a quadratic capacity constraint. We study the polytopes of the concentrator location problems in Section 4. Section 5 is devoted to the branch and cut algorithm and the computational results.

\section{Formulation of QCL}

Let $I$ denote the set of terminal nodes with $|I|=n$ and $K$ denote the set of all directed pairs of nodes. We denote by $T_{i m}$ the amount of traffic between nodes $i$ and $m$ for each $(i, m) \in K$. So $T_{i m}=T_{m i}$ for $(i, m) \in K$. We define $T_{i i}=0$ for all $i \in I$.

Each terminal either receives a concentrator or is assigned to a concentrator node. Let $a_{i}$ be the fixed demand of terminal $i \in I$. If node $j \in I$ becomes a concentrator node, then it has capacity $M$.

Define $A=\{(i, j): i \in I, j \in I \backslash\{j\}\}$. The cost of assigning node $i$ to node $j$ for $(i, j) \in A$ is denoted by $C_{i j}$ and the cost of installing a concentrator at node $i$ is denoted by $C_{i i}$. We define $x_{i j}$ to be 1 if node $i \in I$ is assigned to node $j \in I$ and 0 otherwise. If node $j$ receives a concentrator, then $x_{j j}=1$ and so node $j$ is assigned to itself.

The QCL can be formulated as follows:

$$
\begin{aligned}
& \min \sum_{i \in I} \sum_{j \in I} C_{i j} x_{i j} \\
& \text { s.t. } \sum_{j \in I} x_{i j}=1 \quad \forall i \in I \\
& \quad x_{i j} \leq x_{j j} \quad \forall(i, j) \in A
\end{aligned}
$$




$$
\begin{aligned}
& \sum_{i \in I} a_{i} x_{i j}+\sum_{i \in I} \sum_{m \in I} T_{i m} x_{i j}\left(1-x_{m j}\right) \leq M x_{j j} \quad \forall j \in I \\
& x_{i j} \in\{0,1\} \quad \forall i, j \in I .
\end{aligned}
$$

Constraints (2) and (5) imply that each node $i \in I$ should be assigned to exactly one node. Constraints (3) ensure that if node $i$ is assigned to node $j$, then node $j$ receives a concentrator. If node $j$ receives a concentrator, then the sum of fixed demands of nodes assigned to node $j$ and the amount of traffic between nodes assigned to $j$ and nodes that are assigned to other concentrators cannot exceed the capacity $M$ due to constraints (4). The objective function (1) is the sum of concentrator location and assignment costs and the aim is to minimize this total cost.

To model the capacity constraints which impose a limit on the traffic on the links adjacent at a concentrator node, we take $a_{i}=\sum_{m \in I} T_{i m}$ for all $i \in I$. But all the results of the paper remain valid for $a_{i} \geq \sum_{m \in I} T_{i m}$ for all $i \in I$.

In the sequel, we assume that

1. $a_{i} \geq \sum_{m \in I} T_{i m}$ for all $i \in I$,

2. any two nodes can be assigned together, i.e., $a_{i}+a_{m}+\sum_{l \in I \backslash\{i, m\}}\left(T_{i l}+T_{m l}\right) \leq M$ for all $(i, m) \in A$.

We also consider two variants of QCL based on the capacity constraints.

- The Linear Capacitated Concentrator Location Problem (LCL): If the capacity of a concentrator is defined in terms of the fixed demands of nodes assigned to it, then the capacity constraints become $\sum_{i \in I} a_{i} x_{i j} \leq M x_{j j}$ for $j \in I$. The LCL is a special case of QCL where $T_{i m}=0$ for all $(i, m) \in K$.

- The Uncapacitated Concentrator Location Problem (UCL): If all terminals can be assigned to a single concentrator, i.e., if $\sum_{i \in I} a_{i} \leq M$ then constraints (4) can be removed. The UCL is also a special case of QCL where $\sum_{i \in I} a_{i} \leq M$.

\section{Quadratic knapsack constraint}

In QCL, the capacity constraint is of the form

$$
\sum_{i \in I} a_{i} u_{i}+\sum_{i \in I} \sum_{m \in I} T_{i m} u_{i}\left(1-u_{m}\right) \leq M .
$$

We present five linearizations of the quadratic knapsack constraint (6). The first three linearizations are based on linear knapsack constraints. Define $F_{Q K}=\left\{u \in\{0,1\}^{n}\right.$ : $u$ satisfies (6)\}.

Proposition 1. The point $u \in\{0,1\}^{n}$ is in $F_{Q K}$ if and only if it satisfies inequalities

$$
\sum_{i \in I} a_{i} u_{i}+\sum_{i \in I^{\prime}} \sum_{m \in I \backslash I^{\prime}} T_{i m}\left(u_{i}-u_{m}\right) \leq M
$$

for all $I^{\prime} \subseteq I$ 
Proof: Given $u \in\{0,1\}^{n}$, define $I^{*}=\left\{i \in I: u_{i}=1\right\}$. Then $u$ is in $F_{Q K}$ if and only if $\sum_{i \in I^{*}} a_{i}+\sum_{i \in I^{*}} \sum_{m \in I \backslash I^{*}} T_{i m} \leq M$. This is inequality (7) defined by $I^{*}$. As $I^{*}$ maximizes the left hand side of inequality (7), $u$ satisfies inequality (7) for $I^{*}$ if and only if it satisfies it for all $I^{\prime} \subseteq I$.

Proposition 2. The point $u \in\{0,1\}^{n}$ is in $F_{Q K}$ if and only if it satisfies inequalities

$$
\sum_{i \in I} a_{i} u_{i}+\sum_{(i, m) \in K^{\prime}} T_{i m}\left(u_{i}-u_{m}\right) \leq M
$$

for all $K^{\prime} \subseteq K$

Proof: Given $u \in\{0,1\}^{n}$, define $I^{*}=\left\{i \in I: u_{i}=1\right\}$ and $K^{*}=\{(i, m) \in K: i \in$ $\left.I^{*}, m \in I \backslash I^{*}\right\}$. The left hand side of inequality (8) for $K^{*}$ is $\sum_{i \in I} a_{i} u_{i}+\sum_{(i, m) \in K^{*}} T_{i m}\left(u_{i}-\right.$ $\left.u_{m}\right)=\sum_{i \in I^{*}} a_{i}+\sum_{i \in I^{*}} \sum_{m \in I \backslash I^{*}} T_{i m}$ and $K^{*}$ is a set that maximizes the left hand side of inequality (8).

The third linearization is based on the results in Balas and Mazzola [5]. Define $a_{i}^{\prime}=$ $a_{i}+\sum_{m \in I} T_{i m}$ for all $i \in I$.

Proposition 3. The point $u \in\{0,1\}^{n}$ is in $F_{Q K}$ if and only if it satisfies inequalities

$$
\sum_{i \in I} a_{i}^{\prime} u_{i}-\sum_{(i, m) \in K} T_{i m} u_{\phi(i, m)} \leq M
$$

for all $\phi: K \rightarrow I$ such that $\phi(i, m) \in\{i, m\}$ for all $(i, m) \in K$.

Proof: Given $u \in\{0,1\}^{n}$, define $I^{*}=\left\{i \in I: u_{i}=1\right\}$ and $\phi^{*}(i, m)=i$ if $i \notin I^{*}$ and $m$ otherwise for all pairs $(i, m) \in K$. This implies that $u_{\phi^{*}(i, m)}=1$ if and only if $u_{i}=1$ and $u_{m}=1$. Then $\sum_{i \in I} a_{i}^{\prime} u_{i}-\sum_{(i, m) \in K} T_{i m} u_{\phi^{*}(i, m)}=\sum_{i \in I^{*}}\left(a_{i}^{\prime}-\sum_{m \in I^{*}} T_{i m}\right)=$ $\sum_{i \in I^{*}}\left(a_{i}+\sum_{m \in I \backslash I^{*}} T_{i m}\right)$ and $\phi^{*}$ maximizes the left hand side of inequality (9).

Let $L_{i}$ denote the set of feasible solutions for the LP relaxation of the $i$ th linearization, i.e., $L_{1}=\left\{u \in[0,1]^{n}: u\right.$ satisfies (7) for all $\left.I^{\prime} \subseteq I\right\}, L_{2}=\left\{u \in[0,1]^{n}: u\right.$ satisfies (8) for all $\left.K^{\prime} \subseteq K\right\}$ and $L_{3}=\left\{u \in[0,1]^{n}: u\right.$ satisfies (9) for all $\phi: K \rightarrow I$ such that $\phi(i, m) \in\{i, m\}$ for all $(i, m) \in K\}$.

Proposition 4. $L_{3}=L_{2} \subseteq L_{1}$.

Proof: We first prove that $L_{3}=L_{2}$. For a given $\phi$, let $K^{\prime}=\{(i, m) \in K: \phi(i, m)=m\}$ and for a given $K^{\prime}$ let $\phi(i, m)$ be $i$ if $(i, m) \notin K^{\prime}$ and $m$ otherwise. Inequality (8) defined by $K^{\prime}$ is the same as inequality (9) defined by $\phi$. Now we show that $L_{2} \subseteq L_{1}$. Given $I^{\prime} \subseteq I$, define $K^{\prime}=\left\{(i, m) \in K: i \in I^{\prime}, m \notin I^{\prime}\right\}$. Inequality (7) defined by $I^{\prime}$ is the same as inequality (8) defined by $K^{\prime}$. 
The next two linearizations are based on cover inequalities.

Definition 1. A subset $C \subseteq I$ such that $\sum_{i \in C} a_{i}+\sum_{i \in C} \sum_{m \in I \backslash C} T_{i m}>M$ is called a quadratic cover. A quadratic cover $C$ is called a minimal quadratic cover if no proper subset of $C$ is a quadratic cover.

Proposition 5. If $C \subseteq I$ is a quadratic cover, then the quadratic cover inequality

$$
\sum_{i \in C} u_{i} \leq|C|-1
$$

is valid for $F_{Q K}$.

Proof: $\quad$ As $a_{i} \geq \sum_{m \in I} T_{i m}$ for all $i \in I, F_{Q K}$ is an independence system (see [18]).

Proposition 6. The point $u \in\{0,1\}^{n}$ is in $F_{Q K}$ if and only if it satisfies inequalities (10) for all $C \subseteq I$ such that $C$ is a minimal quadratic cover.

Proof: Given $u \in\{0,1\}^{n}$, define $I^{*}=\left\{i \in I: u_{i}=1\right\}$. If $u$ is not in $F_{Q K}$, then $\sum_{i \in I^{*}} a_{i}+\sum_{i \in I^{*}} \sum_{m \in I \backslash I^{*}} T_{i m}>M$. So $I^{*}$ is a quadratic cover and the corresponding inequality (10) is violated since $\sum_{i \in I^{*}} u_{i}=\left|I^{*}\right|$. If $I^{*}$ is not minimal, there exists a subset of $I^{*}$ which is a minimal cover and for which inequality (10) is violated.

If $u \in\{0,1\}^{n}$ is in $F_{Q K}$ and $C \subseteq I$ is a minimal quadratic cover, $u$ satisfies inequality (10) due to Proposition 5.

Proposition 7. The point $u \in\{0,1\}^{n}$ is in $F_{Q K}$ if and only if it satisfies inequalities (10) for all $C \subseteq I$ such that $C$ is a minimal cover for a knapsack constraint (8) for some $K^{\prime} \subseteq K$.

Proof: Given $u \in\{0,1\}^{n}$, define $I^{*}=\left\{i \in I: u_{i}=1\right\}$ and $K^{*}=\{(i, m) \in K: i \in$ $\left.I^{*}, m \in I \backslash I^{*}\right\}$. The knapsack constraint (8) for $K^{\prime}=K^{*}$ is

$$
\sum_{i \in I^{*}}\left(a_{i}+\sum_{m \in I \backslash I^{*}} T_{i m}\right) u_{i}+\sum_{m \in I \backslash I^{*}}\left(a_{m}-\sum_{i \in I^{*}} T_{i m}\right) u_{m} \leq M
$$

If $u$ is not in $F_{Q K}$, then $\sum_{i \in I^{*}}\left(a_{i}+\sum_{m \in I \backslash I^{*}} T_{i m}\right)>M$ and so $I^{*}$ is a cover for knapsack constraint (8) for $K^{\prime}=K^{*}$. Since $\sum_{i \in I^{*}} u_{i}=\left|I^{*}\right|$, the corresponding cover inequality (10) is violated. Again, if $I^{*}$ is not minimal, there exists a subset of $I^{*}$ which is a minimal cover and the corresponding cover inequality is violated.

Let $K^{\prime} \subseteq K$. As set $F_{Q K}$ is a subset of the set of vectors $u \in\{0,1\}^{n}$ which satisfy inequality (8) for $K^{\prime}$, all valid inequalities for the latter set are also valid for $F_{Q K}$. This proves that if $u \in F_{Q K}$, it satisfies inequalities (10) for all covers for the knapsack constraint (8). 
Let $L_{4}$ and $L_{5}$ denote the set of feasible solutions of the LP relaxations of the last two linearizations, i.e., $L_{4}=\left\{u \in[0,1]^{n}: u\right.$ satisfies inequalities (10) for all $C \subseteq I$ such that $C$ is a minimal quadratic cover $\}$ and $L_{5}=\left\{u \in[0,1]^{n}: u\right.$ satisfies inequalities (10) for all $C \subseteq I$ such that $C$ is a minimal cover for a knapsack constraint (8) for some $K^{\prime} \subseteq K$ \}.

Proposition 8. $\quad L_{4}=L_{5}$.

Proof: For a given $u$, to check if there exists a cover $C$ for a knapsack constraint (8) defined by some $K^{\prime} \subseteq K$ for which the cover inequality is violated, we solve the following problem:

$$
\begin{aligned}
\min & \sum_{i \in I}\left(1-u_{i}\right) v_{i} \\
\text { s.t. } & \sum_{i \in I}\left(a_{i}+\sum_{m \in I} T_{i m} y_{i m}-\sum_{m \in I} T_{m i} y_{m i}\right) v_{i}>M \\
& v_{i} \in\{0,1\} \quad \forall i \in I \\
& y_{i m} \in\{0,1\} \quad \forall(i, m) \in K
\end{aligned}
$$

where $v_{i}$ is 1 if $i \in C$ and 0 otherwise and $y_{i m}=1$ if $(i, m) \in K^{\prime}$ and 0 otherwise. For a given $v$, an optimal $y$ can be found by taking $y_{i m}$ to be 1 if $v_{i}=1$ and $v_{m}=0$ and 0 otherwise. Then the left hand side of the constraint is $\sum_{i \in I}\left[a_{i}+\sum_{m \in I} T_{i m} v_{i}\left(1-v_{m}\right)-\right.$ $\left.\sum_{m \in I} T_{m i} v_{m}\left(1-v_{i}\right)\right] v_{i}=\sum_{i \in I} a_{i} v_{i}+\sum_{i \in I} \sum_{m \in I} T_{i m} v_{i}\left(1-v_{m}\right)$ since $v_{i}^{2}=v_{i}$ for all $i \in I$. This is the problem we solve to find a violated quadratic cover inequality.

We cannot compare $L_{2}$ and $L_{5}$. It is well known that $L_{5}$ is not included in $L_{2}$. In general $L_{2}$ is not included in $L_{5}$ either.

Example 1. Let $I=\{1, \ldots, 5\}, M=80, T_{i m}=10$ for all $(i, m) \in K$ and $a_{i}=40$ for all $i \in I$. Consider the fractional solution $u$ where $u_{1}=0.9$ and $u_{i}=0.1$ for $i \neq 1$. It is not in $L_{2}$ since inequality (8) is violated for $K^{\prime}=\{(1,2),(1,3),(1,4),(1,5)\}$. However, there is no violated cover inequality since $\left(1-u_{i}\right)+\left(1-u_{m}\right) \geq 1$ for any two items $i$ and $m$ and no one item subset is a cover.

Define $P_{Q K}=\operatorname{conv}\left(F_{Q K}\right)$. As $a_{i}+\sum_{m \in I} T_{i m} \leq M$ for all $i \in I$, polytope $P_{Q K}$ has dimension $n$ and inequalities $u_{i} \geq 0$ for $i \in I$ are the trivial facets of $P_{Q K}$.

Consider the linear knapsack set $\left\{u \in\{0,1\}^{n}: \sum_{i \in I} a_{i} u_{i} \leq M\right\}$. Let $C \subseteq I$ be a cover. The cover inequality $\sum_{i \in C} u_{i} \leq|C|-1$ defines a facet of $\operatorname{conv}\left(\left\{u \in\{0,1\}^{n}: \sum_{i \in I} a_{i} u_{i} \leq\right.\right.$ $\left.M, u_{i}=0 \forall i \in I \backslash C\right\}$ ) if and only if $C$ is minimal (see [4, 13] and [20]). Here we have a very similar result for quadratic covers.

Proposition 9. If $C \subseteq I$ is a minimal quadratic cover, then quadratic cover inequality (10) is facet defining for $\operatorname{conv}\left(F_{Q K} \cap\left\{u \in\{0,1\}^{n}: u_{i}=0 \forall i \in I \backslash C\right\}\right)$. 
Yaman [21] presents branch and bound algorithms to solve the quadratic integer programming problems associated with the separation of the quadratic cover inequalities and the computation of lifting coefficients.

\section{Polyhedral analysis}

We eliminate the $x_{j j}$ variables by substituting $x_{j j}=1-\sum_{i \in I \backslash\{j\}} x_{j i}$ for all $j \in I$ (see [3]). Using the fact that $x_{i j} x_{j j}=x_{i j}$ for all $i \in I$ and $j \in I \backslash\{j\}$, the QCL can be modeled as follows:

$$
\begin{array}{ccc}
\min \sum_{i \in I} \sum_{j \in I \backslash\{i\}} C_{i j} x_{i j}+\sum_{i \in I} C_{i i}\left(1-\sum_{j \in I \backslash\{i\}} x_{i j}\right) & \\
\text { s.t. } x_{i j}+\sum_{k \in I \backslash\{j\}} x_{j k} \leq 1 & \forall(i, j) \in A \\
\qquad \sum_{i \in I \backslash\{j\}}\left(a_{i}-T_{i j}\right) x_{i j}+\sum_{i \in I \backslash\{j\}} \sum_{m \in I \backslash\{j\}} T_{i m} x_{i j}\left(1-x_{m j}\right) & \\
\leq\left(M-a_{j}-\sum_{i \in I \backslash\{j\}} T_{i j}\right)\left(1-\sum_{k \in I \backslash\{j\}} x_{j k}\right) & \forall j \in I \\
x_{i j} \in\{0,1\} & \forall(i, j) \in A .
\end{array}
$$

Let $F_{Q}=\left\{x \in\{0,1\}^{n(n-1)}: x\right.$ satisfies (11) and (12) $\}$ and $P_{Q}=\operatorname{conv}\left(F_{Q}\right)$. The LCL polytope $P_{L}$ is obtained by setting $T_{i m}=0$ for all $(i, m) \in K$ and the UCL polytope $P_{U}$ is obtained by taking $M=\sum_{i \in I} a_{i}$. Polytope $P_{U}$ is a special stable set polytope.

For $(i, j) \in A$, define the unit vector $e^{i j}$ such that $e_{i j}^{i j}=1$ and $e_{k l}^{i j}=0$ for all $(k, l) \in$ $A \backslash\{(i, j)\}$.

We say node $i \in I$ is open if $i$ receives a concentrator and it is free if in addition, no other node is assigned to $i$.

Proposition 10. Polytope $P_{Q}$ is full dimensional, i.e., $\operatorname{dim}\left(P_{Q}\right)=n(n-1)$.

Proof: Consider points $x=0$ and $e^{i j}$ for each $(i, j) \in A$.

Proposition 11. The constraint $x_{i j} \geq 0$ defines a facet of $P_{Q}$ for $(i, j) \in A$.

Proof: Consider points $x=0$ and $e^{k l}$ for each $(k, l) \in A \backslash\{(i, j)\}$.

If $n=3$ and $a_{1}+a_{2}+a_{3}>M$, then polytopes $P_{Q}$ and $P_{L}$ are defined by the inequality $x_{12}+x_{13}+x_{21}+x_{23}+x_{31}+x_{32} \leq 1$ and the nonnegativity constraints. So in the sequel, for capacitated problems, we assume that $n \geq 4$.

Proposition 12. For $(i, j) \in A$, inequality $(11)$ defines a facet of $P_{Q}$ if and only if $\{i, j, k\}$ is not a quadratic cover for all $k \in I \backslash\{i, j\}$. 
Proof: Assume that $\{i, j, k\}$ is not a quadratic cover for all $k \in I \backslash\{i, j\}$. We give $n(n-1)$ affinely independent points in $P_{Q}$ that satisfy (11) at equality:

1. $e^{i j}+e^{k t}$ for $(k, t) \in A$ such that $k, t \in I \backslash\{i, j\}$

2. $e^{j i}+e^{k i}$ for $k \in I \backslash\{i, j\}$

3. $e^{i j}+e^{k j}$ for $k \in I \backslash\{i, j\}$

4. $e^{j k}+e^{i k}$ for $k \in I \backslash\{i, j\}$

5. $e^{i j}$

6. $e^{j i}$

7. $e^{j k}$ for $k \in I \backslash\{i, j\}$.

If there exists a node $k \in I \backslash\{i, j\}$ such that $\{i, j, k\}$ is a quadratic cover, then inequality (11) can be strengthened as follows: $x_{i j}+x_{k j}+\sum_{l \in I \backslash\{j\}} x_{j l} \leq 1$.

In the sequel, we present facet defining inequalities for $P_{Q}$ using its relation to knapsack, stable set and facility location polytopes.

\subsection{Knapsack based inequalities}

We show how the facet defining inequalities of knapsack polytopes can be lifted to define facets of location polytopes.

Proposition 13. If inequality $\sum_{i \in I \backslash\{j\}} \alpha_{i} u_{i} \leq \alpha_{0}$ is a valid inequality for the quadratic knapsack polytope $P_{j}=\operatorname{conv}\left(\left\{u \in\{0,1\}^{n-1}: \sum_{i \in I \backslash\{j\}}\left(a_{i}-T_{i j}\right) u_{i}+\sum_{i \in I \backslash\{j\}} \sum_{m \in I \backslash j\}}\right.\right.$ $\left.\left.T_{i m} u_{i}\left(1-u_{m}\right) \leq M-a_{j}-\sum_{i \in I} T_{i j}\right\}\right)$, then inequality

$$
\sum_{i \in I \backslash\{j\}} \alpha_{i} x_{i j}+\sum_{i \in I \backslash\{j\}} \alpha_{0} x_{j i} \leq \alpha_{0}
$$

is a valid inequality for $P_{Q}$.

Proof: If $x_{j i}=0$ for all $i \in I \backslash\{j\}$, then knapsack inequality $\sum_{i \in I \backslash j\}}\left(a_{i}-T_{i j}\right) u_{i}+$ $\sum_{i \in I \backslash j\}} \sum_{m \in I \backslash\{j\}} T_{i m} u_{i}\left(1-u_{m}\right) \leq M-a_{j}-\sum_{i \in I} T_{i j}$ is equivalent to capacity constraint (12) for node $j$. If $x_{j l}=1$ for some $l \in I \backslash\{j\}$, then $x_{i j}=0$ for all $i \in I \backslash\{j\}$ and $x_{j i}=0$ for all $i \in I \backslash\{j, l\}$.

Theorem 1. If inequality $\sum_{i \in I \backslash\{j\}} \alpha_{i} u_{i} \leq \alpha_{0}$ is facet defining for polytope $P_{j}$, then inequality (14) is facet defining for $P_{Q}$.

Proof: If inequality $\sum_{i \in I \backslash\{j\}} \alpha_{i} u_{i} \leq \alpha_{0}$ is facet defining for polytope $P_{j}$, then inequality $\sum_{i \in I \backslash\{j\}} \alpha_{i} x_{i j} \leq \alpha_{0}$ is facet defining for $\operatorname{conv}\left(F_{Q} \cap\left\{x: x_{k l}=0 \forall k \in I, l \in I \backslash\{k, j\}\right\}\right)$. We lift all variables fixed to 0 sequentially. We start with variables $x_{j k}$ for $k \in I \backslash\{j\}$. If $x_{j k}=1$, then $x_{i j}=0$ for all $i \in I \backslash\{j\}$ and $x_{j i}=0$ for all $i \in I \backslash\{j, k\}$. Hence the coefficient of $x_{j k}$ is $\alpha_{0}$. Now we lift the variables $x_{k l}$ where $k \in I \backslash\{j\}$ and $l \in I \backslash\{k, j\}$. If $x_{k l}=1$, then as $e^{j m}$ for some $m \in I \backslash\{k, l, j\}$ is feasible, the lifting coefficient of $x_{k l}$ is 0 . 


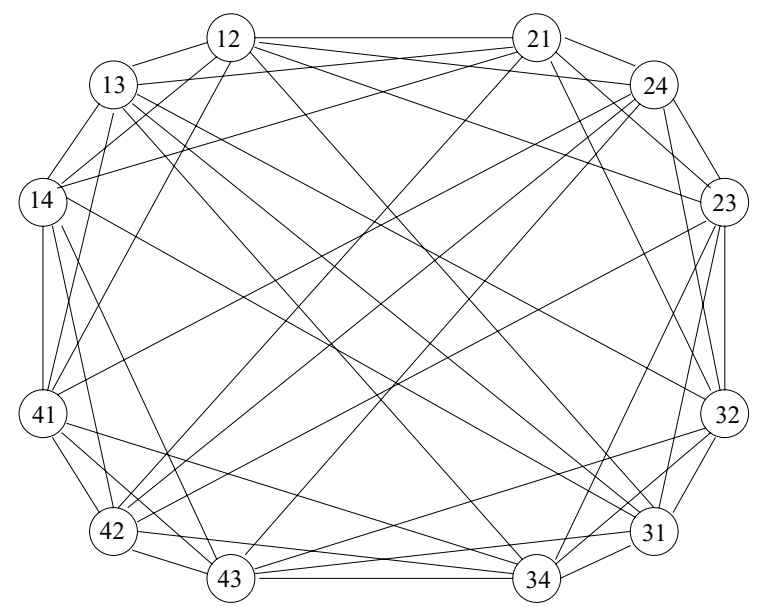

Figure 1. Conflict graph $G_{C}$ for $n=4$

\subsection{Stable set based inequalities}

As UCL is a special stable set problem and a relaxation of QCL, valid inequalities for the stable set polytope are also valid for $P_{Q}$.

Consider the conflict graph $G_{C}$ associated with UCL and defined as follows. For each arc in the set $A$, add a vertex in $G_{C}$. There is an edge between two vertices of $G_{C}$ if and only if there exists a constraint (11) where the corresponding variables appear together. Figure 1 depicts $G_{C}$ for $n=4$ where arc (vertex in $\left.G_{C}\right)(i, j)$ is written as $i j$ for short.

Proposition 14. A maximal clique in $G_{C}$ is either $\{(i, j),(j, l),(l, i)\}$ for $i \neq j \neq l$ or $\{(m, i)\} \cup\{(i, j): \forall j \in I \backslash\{i\}\}$.

Proof: In $G_{C}$, there is an edge between vertices $(i, j)$ and $(k, l)$ if and only if (i) $i=k$ and $j \neq l$ or (ii) $i=l$ or (iii) $j=k$. A clique that contains $(i, j)$ and $(i, l)$ for $j \neq l$ can contain at most one vertex $(m, i)$ and any number of vertices of the form $(i, m)$. A maximal such clique is $\{(m, i)\} \cup\{(i, j): \forall j \in I \backslash\{i\}\}$.

A clique where no node is repeated as the tail, i.e., if $(i, j)$ is in the clique, no other vertex of the form $(i, m)$ can be in the clique, contains two vertices $(i, j)$ and $(j, l)$ with $i \neq j \neq l$. In this case, the only vertex that can appear in such a clique is $(l, i)$.

So facet defining clique inequalities for $P_{U}$ are constraints (11) and triangle inequalities $x_{i j}+x_{j l}+x_{l i} \leq 1$ for $i \neq j \neq l$.

When $n=3$, constraints (11), nonnegativity constraints and the two triangle inequalities describe polytope $P_{U}$ (see [21]).

4.2.1. $W-2$ Inequalities. The $W-2$ inequalities are introduced by Avella and Sassano [3] for the p-median polytope. They correspond to known families of valid inequalities for 
the stable set polytope and thus for $P_{U}$.

Theorem 2. Let $W \subseteq I$ with $|W| \geq 4$ and $H \subset A(W)=\{(i, j) \in A: i \in W, j \in W\}$ be such that $|H \cap\{(i, j) \in A(W)\}|=1$ for all $j \in W$. Define $W_{j}=\{i \in W \backslash\{j\}:(i, j) \notin H\}$ and $U=\left\{i \in W: i \in W_{j} \forall j \in W \backslash\{i\}\right\}$. Let $R=A(W) \backslash H \cup\{(i, j): i \in U, j \in I \backslash W\}$. The $W-2$ inequality

$$
\sum_{(i, j) \in R} x_{i j} \leq|W|-2
$$

defines a facet of $P^{\prime}=\operatorname{conv}\left(F_{Q} \cap\left\{x \in\{0,1\}^{n(n-1)}: x_{i u}=0 \forall i \in I \backslash W, u \in U\right.\right.$ and $x_{i j}=$ $0 \forall(i, j) \in H\})$, if $|U| \leq|W|-3$ and $W_{j} \cup\{j\}$ is not a quadratic cover for all $j \in W$.

Proof: Assume that $|W| \geq 4,|U| \leq|W|-3$ and $W_{j} \cup\{j\}$ is not a quadratic cover for all $j \in W$. Let $A_{0}=\{(i, u) \in A: i \in I \backslash W, u \in U\}$. Define $P_{f}=\left\{x \in P^{\prime}\right.$ : $\left.\sum_{(i, j) \in R} x_{i j}=|W|-2\right\}$. Assume that all $x \in P_{f}$ satisfy $\sum_{(i, j) \in A \backslash\left(A_{0} \cup H\right)} \alpha_{i j} x_{i j}=\alpha_{0}$. Define $\left\{m_{j}\right\}=W \backslash\left(W_{j} \cup\{j\}\right)$ for all $j \in W$.

Consider $(m, l) \in A$ such that $m \in I \backslash W$ and $l \in I \backslash W$. As both $x=\sum_{i \in W_{j}} e^{i j}$ for some $j \in W$ and $x+e^{m l}$ are in $P_{f}$, we have that $\alpha_{m l}=0$.

Consider $l \in W \backslash U$ and $m \in I \backslash W$. There exists a node $j \in W$ such that $m_{j}=l$. Define $x=\sum_{i \in W_{j}} e^{i j}, x^{\prime}=x+e^{l m}$ and $x^{\prime \prime}=x+e^{m l}$. As $x, x^{\prime}$ and $x^{\prime \prime}$ are in $P_{f}, \alpha_{l m}=\alpha_{m l}=0$.

Consider $(u, j) \in A$ such that $u \in U$ and $j \in W$. As both $x=\sum_{i \in W_{j}} e^{i j}$ and $x-e^{u j}+e^{u l}$ for $l \in I \backslash W$ are in $P_{f}$, we have that $\alpha_{u j}=\alpha_{u l}$. This implies that $\alpha_{u j}=\alpha_{u}$ for all $j \in I \backslash\{u\}$.

Let $(i, u) \in A(W) \backslash H$ such that $i \in W \backslash U$ and $u \in U$. There exists a node $l \in W$ such that $m_{l}=i$. Both $x=\sum_{t \in W_{l} \backslash\{u\}} e^{t l}+e^{i u}$ and $x-e^{i u}+e^{u i}$ are in $P_{f}$. So $\alpha_{i u}=\alpha_{u i}=\alpha_{u}$ for all $i \in W \backslash U, u \in U$.

Let $u$ and $v$ be two different nodes in $U$. Both $\sum_{i \in W_{u}} e^{i u}$ and $\sum_{i \in W_{v}} e^{i v}$ are in $P_{f}$. As $\sum_{i \in W_{u}} \alpha_{i u}=\sum_{i \in W_{u} \backslash U} \alpha_{u}+\sum_{i \in U \backslash\{u\}} \alpha_{i}=(|W|-|U|-1) \alpha_{u}+\sum_{i \in U} \alpha_{i}-\alpha_{u}=$ $(|W|-|U|-2) \alpha_{u}$ and similarly $\sum_{i \in W_{v}} \alpha_{i v}=(|W|-|U|-2) \alpha_{v}, \alpha_{u}=\alpha$ for all $u \in U$.

Consider $(i, j) \in A$ such that $j \in W \backslash U$ and $i \in W_{j} \backslash U$. If there exists a node $u \in U$ such that $m_{j} \neq m_{u}$ and $i \neq m_{u}$, then $x=\sum_{l \in W_{j} \backslash\{u\}} e^{l j}+e^{m_{j} u}$ and $x-e^{i j}+e^{i u}$ are in $P_{f}$. So $\alpha_{i j}=\alpha_{i u}=\alpha$.

If there exists a node $u$ such that $i=m_{u}$, then consider $x=\sum_{l \in W_{u}} e^{l u}$ and $x^{\prime}=$ $x-e^{j u}+e^{i j}$. We have $\alpha_{i j}=\alpha$ as both $x$ and $x^{\prime}$ are in $P_{f}$ and $\sum_{l \in W_{u}} \alpha_{l u}=(|W|-2) \alpha=$ $\sum_{l \in W_{u} \backslash\{j\}} \alpha_{l u}+\alpha_{i j}=(|W|-3) \alpha+\alpha_{i j}$.

If $m_{u}=m_{j}$ for all nodes $u \in U$ and $m_{m_{j}} \neq i$, then $\alpha_{i m_{j}}=\alpha$. As both $x=\sum_{t \in W_{j}} e^{t j}$ and $x-e^{i j}+e^{i m_{j}}$ are in $P_{f}$, we have that $\alpha_{i j}=\alpha_{i m_{j}}=\alpha$.

If $m_{u}=m_{j}$ for all nodes $u \in U$ and $m_{m_{j}}=i$, then $\alpha_{k m_{j}}=\alpha$ for all $k \in W_{m_{j}}$ and $\alpha_{k j}=\alpha$ for all $k \in W_{j} \backslash\{i\}$. The points $\sum_{t \in W_{j}} e^{t j}$ and $\sum_{t \in W_{m_{j}}} e^{t m_{j}}$ are in $P_{f}$. So $\alpha_{i j}+(|W|-3) \alpha=(|W|-2) \alpha, \alpha_{i j}=\alpha$ and $\alpha_{0}=|W-2| \alpha$.

Figure 2 depicts a $W-2$ structure. In this example, $I=\{1,2, \ldots, 9\}, W=\{1,2,3,4\}$ and $U=\{4\}$. The dashed line arcs form the set $H=\{(1,4),(2,3),(3,1),(3,2)\}$. The corresponding $W-2$ inequality is $x_{12}+x_{13}+x_{21}+x_{24}+x_{34}+\sum_{i \in I \backslash\{4\}} x_{4 i} \leq 2$. 


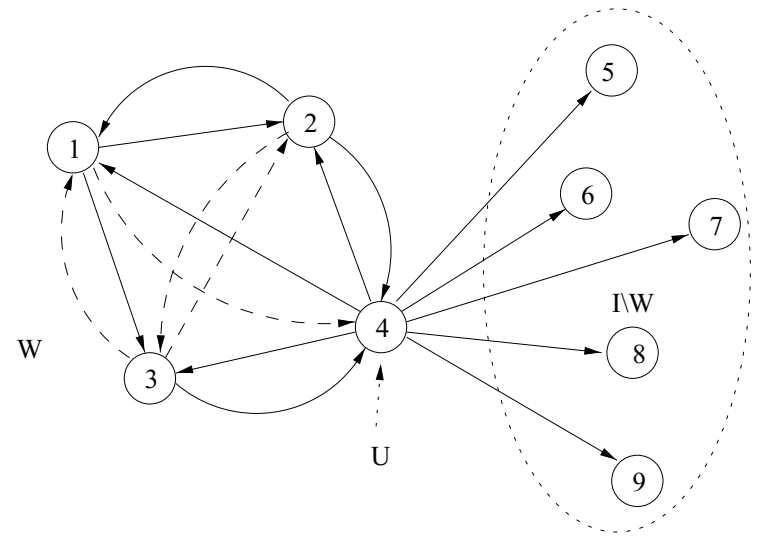

Figure 2. A $W-2$ structure

Starting from $W-2$ inequality (15) and applying sequential lifting, we can obtain a facet defining inequality for $P_{Q}$ of the form

$$
\sum_{(i, j) \in R} x_{i j}+\sum_{(i, u) \in A_{0}} \pi_{i u} x_{i u}+\sum_{(i, j) \in H} \alpha_{i j} x_{i j} \leq|W|-2 .
$$

We first show that independently of the lifting sequence, the optimal lifting coefficients are either 0 or 1 . For $(i, j) \in A_{0} \cup H$, define $A_{i j} \subseteq A_{0} \backslash\{(i, j)\}$ and $H_{i j} \subseteq H \backslash\{(i, j)\}$ to be the set of $\operatorname{arcs}(k, l)$ in $A_{0}$ and in $H$ respectively such that $x_{k l}$ is lifted before $x_{i j}$. Define also $F_{i j}=F_{Q} \cap\left\{x \in\{0,1\}^{n(n-1)}: x_{k l}=0 \forall(k, l) \in\left(\left(H \backslash H_{i j}\right) \cup\left(A_{0} \backslash A_{i j}\right)\right) \backslash\{(i, j)\}, x_{i j}=1\right\}$.

Proposition 15. The coefficient $\pi_{l u} \in\{0,1\}$ for all $(l, u) \in A_{0}$.

Proof: The coefficient $\pi_{l u}$ can be computed as

$$
\pi_{l u}=|W|-2-\max _{x \in F_{l u}}\left(\sum_{(i, j) \in R} x_{i j}+\sum_{(r, v) \in A_{l u}} \pi_{r v} x_{r v}+\sum_{(k, l) \in H_{l u}} \alpha_{k l} x_{k l}\right) .
$$

The point $x=\sum_{i \in W_{j} \backslash\{u\}} e^{i j}+e^{l u}$ for some $j \in W \backslash\{u\}$ is in $F_{l u}$ and it satisfies $\sum_{(i, j) \in R} x_{i j}=$ $|W|-3$. So $\pi_{l u} \leq 1$. As $\pi_{l u} \geq 0$ and it takes only integer values, $\pi_{l u} \in\{0,1\}$.

Proposition 16. The coefficient $\alpha_{i j} \in\{0,1\}$ for all $(i, j) \in H$.

Proof: Given $(i, j)$ in $H$, we can compute the coefficient $\alpha_{i j}$ as

$$
\alpha_{i j}=|W|-2-\max _{x \in F_{i j}}\left(\sum_{(k, l) \in R} x_{k l}+\sum_{(r, v) \in A_{i j}} \pi_{r v} x_{r v}+\sum_{(k, l) \in H_{i j}} \alpha_{k l} x_{k l}\right) .
$$


If $|W \backslash U| \geq 4$ or $j \in U$, then there exist two nodes $l$ and $k$ in $W \backslash(U \cup\{i, j\})$. As there exists at least one node $t \in W$ such that $m_{t}=k,\{i, j, l\}$ is not a quadratic cover. We can show that $\{i, j, k\}$ is not a quadratic cover similarly. There exists at least one node $t$ in $W \backslash\{i, j\}$ such that $m_{t}=l$ or $m_{t}=k$. If $m_{t}=l$, then consider $x=e^{i j}+e^{l j}+\sum_{v \in W \backslash\{i, j, l, t\}} e^{v t}$. If $m_{t}=k$, then consider $x=e^{i j}+e^{k j}+\sum_{v \in W \backslash\{i, j, k, t\}} e^{v t}$. In both cases, $x$ is in $F_{i j}$ and satisfies $\sum_{(k, l) \in R} x_{k l}=|W|-3$. So, we have that $\alpha_{i j} \leq 1$.

If $|W \backslash U|=3$ and $W \backslash U=\{i, j, l\}, m_{l}$ is either $i$ or $j$. Since $x=e^{i j}+\sum_{t \in U} e^{t l}$ is in $F_{i j}$ and $\sum_{(k, l) \in R} x_{k l}=|U|=|W|-3, \alpha_{i j} \leq 1$.

Proposition 17. Let $(i, j) \in H, A_{i j} \subseteq A_{0}$ and $H_{i j} \subseteq H \backslash\{(i, j)\}$. Assume that inequality $\sum_{(k, l) \in R} x_{k l}+\sum_{(k, l) \in H_{i j}} \alpha_{k l} x_{k l} \leq|W|-2$ is facet defining for conv $\left(F_{Q} \cap\left\{x \in\{0,1\}^{n(n-1)}\right.\right.$ : $\left.\left.x_{k l}=0 \forall(k, l) \in\left(A_{0} \backslash A_{i j}\right) \cup\left(H \backslash H_{i j}\right)\right\}\right)$. Then, the optimal lifting coefficient for $x_{i j}, \alpha_{i j}$, is 1 if there does not exist a set $U^{\prime} \subseteq(U \backslash\{j\})$ such that $(i) W \backslash U^{\prime}$ is not a quadratic cover and (ii) the nodes in $U^{\prime}$ can be assigned to nodes in $I \backslash W$ and is 0 otherwise.

Proof: If there exists a set $U^{\prime} \subseteq(U \backslash\{j\})$ which satisfies conditions (i) and (ii), then clearly there exists $x \in F_{i j}$ with $\sum_{(k, l) \in R} x_{k l}=|W|-2$. So $\alpha_{i j}=0$.

Assume that there does not exist a set $U^{\prime} \subseteq(U \backslash\{j\})$ which satisfies conditions (i) and (ii) and that there exists an $x \in F_{i j}$ with $\sum_{(k, l) \in R} x_{k l}+\sum_{(k, l) \in H_{i j}} \alpha_{k l} x_{k l}=|W|-2$. If $p$ nodes are open in $x$, then $\sum_{(k, l) \in R} x_{k l}+\sum_{(k, l) \in H_{i j}} \alpha_{k l} x_{k l} \leq|W|-p-1$. So $p=1$ and node $j$ is the only open node. As there is no set $U^{\prime} \subseteq(U \backslash\{j\})$ which satisfies conditions (i) and (ii), $\sum_{(k, l) \in R} x_{k l}+\sum_{(k, l) \in H_{i j}} \alpha_{k l} x_{k l} \leq|W|-3$. So $\alpha_{i j}=1$.

Coefficients $\alpha_{i j}$ 's do not depend on the lifting sequence on set $H$. Moreover $\alpha_{i j}=\alpha$ for all $(i, j) \in H$ with $j \in W \backslash U$ and if $\alpha=1$, then $\alpha_{i j}=1$ for all $(i, j) \in H$.

Proposition 18. Let $(l, u) \in A_{0}, A_{l u} \subseteq A_{0} \backslash\{(l, u)\}$ and $H_{l u} \subseteq H$. Assume that inequality $\sum_{(i, j) \in R} x_{i j}+\sum_{(r, v) \in A_{l u}} \pi_{r v} x_{r v} \leq|W|-2$ is facet defining for conv $\left(F_{Q} \cap\left\{x \in\{0,1\}^{n(n-1)}\right.\right.$ : $\left.\left.x_{i j}=0 \forall(i, j) \in\left(A_{0} \backslash A_{l u}\right) \cup\left(H \backslash H_{l u}\right)\right\}\right)$. Then, the optimal lifting coefficient for $x_{l u}, \pi_{l u}$, is 1 if (i) $\{l, u, j\}$ is a quadratic cover for all $j \in W_{u} \backslash U$ and (ii) $\{l, r, u\}$ is a quadratic cover for all $r \in I \backslash W$ such that $(r, u) \in A_{l u}$ and $\pi_{r u}=1$ and is 0 otherwise.

Proof: If there exists a node $j \in W_{u} \backslash U$ such that $\{l, u, j\}$ is not a quadratic cover, then as $x=e^{l u}+e^{j u}+\sum_{k \in W_{t} \backslash\{u\}} e^{k t}$ where $t \in W$ is such that $m_{t}=j$ is in $F_{l u}$ and $\sum_{(i, j) \in R} x_{i j}=|W|-2$, we have that $\pi_{l u}=0$.

If there exists a node $r \in I \backslash W$ such that $(r, u) \in A_{l u}, \pi_{r u}=1$ and $\{l, r, u\}$ is not a quadratic cover, then $x=e^{l u}+e^{r u}+\sum_{k \in W_{t} \backslash\{u\}} e^{k t}$ where $t \in W \backslash\{u\}$ is in $F_{l u}$ and $\sum_{(i, j) \in R} x_{i j}+\sum_{(r, v) \in A_{l u}} \pi_{r v} x_{r v}=|W|-2$. So $\pi_{l u}=0$.

Assume that conditions (i) and (ii) are satisfied for arc $(l, u) \in A_{0}$. We prove that $\pi_{l u}=1$ by induction on the lifting sequence. Assume that $x_{l u}$ is the first variable to lift and that there exists a point $x \in F_{l u}$ such that $\sum_{(i, j) \in R} x_{i j}=|W|-2$. If there are $p$ nodes that are open in $x$, then $\sum_{(i, j) \in R} x_{i j} \leq|W|-p$. So we should have $p \leq 2$. If node $u$ is the only open node, condition (i) implies that nodes in $W_{u} \backslash U$ cannot be assigned to node $u$. So $\sum_{(i, j) \in R} x_{i j} \leq|U|-1 \leq|W|-4$. If $p=2$ and $q$ is the other open node, then as $m_{q}$ cannot be assigned to node $u$, we have $\sum_{(i, j) \in R} x_{i j} \leq|W|-3$. So $x_{l u}$ has coefficient 1 . 


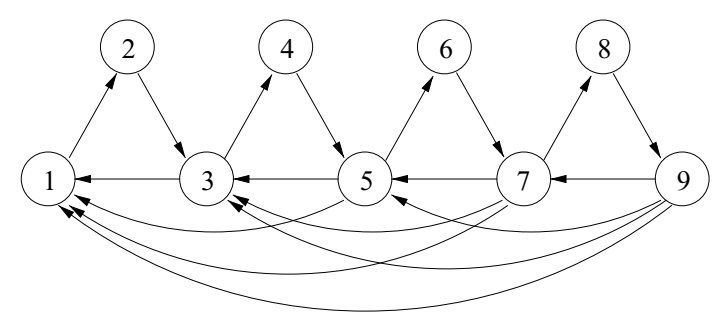

Figure 3. A 4-triangle

Now suppose we lift $x_{l u}$ and all variables $x_{r v}$ with $(r, v) \in A_{l u}$ are lifted as described in the proposition. Assume that there exists a point $x \in F_{l u}$ such that $\sum_{(i, j) \in R} x_{i j}+$ $\sum_{(r, v) \in A_{l u}} \pi_{r v} x_{r v}=|W|-2$. If there are $p$ nodes open in $x$, then $\sum_{(i, j) \in R} x_{i j} \leq|W|-p$. So we should have $\sum_{(r, v) \in A_{l u}} \pi_{r v} x_{r v} \geq p-2$. Conditions (i) and (ii) imply that if $x_{r v}=1$ for some $r \in I \backslash(W \cup\{l\})$ and $v \in U$ with $\pi_{r v}=1$, no other node in $I \backslash U$ whose corresponding assignment variable has coefficient 1 can be assigned to node $v$. Therefore we have at least $p-2$ nodes open in $U \backslash\{u\}$ and either the nodes that are not open in $W \backslash U$ are assigned to the remaining open node, say node $q$ or some node in $I \backslash(W \cup\{l\})$ is assigned to node $q$. As there are at least two nodes not open in $W \backslash U$, we should have all these nodes assigned to node $q$. But as $m_{q}$ cannot be assigned to nodes $q$ and $u$, we have that $\sum_{(i, j) \in R} x_{i j}+\sum_{(r, v) \in A_{l u}} \pi_{r v} x_{r v} \leq|W|-3$. So $\pi_{l u}=1$.

Lifting coefficient $\pi_{l u}$ for $(l, u) \in A_{0}$ depends on lifting coefficients $\pi_{r u}$ for $(r, u) \in A_{l u}$ but is independent of $\pi_{r v}$ with $v \neq u$ and $(r, v) \in A_{l u}$. It can be computed in $O(n)$ time in case of linear capacities and in $O\left(n^{2}\right)$ time in case of quadratic capacities.

If $|W|=3$ then the $W-2$ inequality is either a clique inequality (11) or a triangle inequality which is $x_{i j}+x_{j l}+x_{l i} \leq 1$.

\subsection{2. $k$-triangle inequalities.}

Proposition 19. Let $I^{\prime} \subseteq I$ with $\left|I^{\prime}\right|=2 k+1$ for some $k \geq 1$. Without loss of generality, we number the nodes in $I^{\prime}$ from 1 to $2 k+1$. The arc set $A^{\prime}$ consists of all arcs $(i, i+1)$ for $i=1, . ., 2 k$ and all arcs of the form $(j, l)$ where $j \in I^{\prime}$ and $l \in I^{\prime}$ are both odd and $j>l$. Then the k-triangle inequality

$$
\sum_{(i, j) \in A^{\prime}} x_{i j} \leq k
$$

is valid for $P_{Q}$.

Figure 3 depicts a 4-triangle.

Proof: We prove that the k-triangle inequality (16) is valid for $P_{Q}$ by induction on $k$. When $k=1$, inequality (16) is a triangle inequality. Now assume that inequality (16) is valid for 
$P_{Q}$ for all $k<k^{\prime}$ and that there exists $x \in F_{Q}$ such that $\sum_{(i, j) \in A^{\prime}} x_{i j} \geq k^{\prime}+1$. So $x_{2 k^{\prime}-1,2 k^{\prime}}+$ $x_{2 k^{\prime}, 2 k^{\prime}+1}+\sum_{\left(2 k^{\prime}+1, j\right) \in A^{\prime}} x_{2 k^{\prime}+1, j} \geq 2$. This is possible only if $\sum_{\left(2 k^{\prime}+1, j\right) \in A^{\prime}} x_{2 k^{\prime}+1, j}=1$ and $x_{2 k^{\prime}-1,2 k^{\prime}}=1$ since $\sum_{\left(2 k^{\prime}+1, j\right) \in A^{\prime}} x_{2 k^{\prime}+1, j}+x_{2 k^{\prime}, 2 k^{\prime}+1} \leq 1$ and $x_{2 k^{\prime}-1,2 k^{\prime}}+x_{2 k^{\prime}, 2 k^{\prime}+1} \leq 1$ because of the constraints (11). Hence, $x_{2 k^{\prime}-1,2 k^{\prime}}+x_{2 k^{\prime}, 2 k^{\prime}+1}+\sum_{\left(2 k^{\prime}+1, j\right) \in A^{\prime}} x_{2 k^{\prime}+1, j} \leq 2$. So $x$ should satisfy $\sum_{(i, j) \in A_{-}} x_{i j}=k^{\prime}-1$ where $A_{-}$is the set of arcs for the first $k^{\prime}-1$ triangles.

The clique constraints (11) imply that $\sum_{\left(2 k^{\prime}-1, j\right) \in A^{\prime}} x_{2 k^{\prime}-1, j}=0$ and that $x_{2 k^{\prime}-2,2 k^{\prime}-1}=0$. Moreover since $\sum_{(i, j) \in A_{-}^{\prime}} x_{i j} \leq k^{\prime}-2$ where $A_{-}^{\prime}$ is the set of arcs for the first $k^{\prime}-2$ triangles, we should have $x_{2 k^{\prime}-3,2 k^{\prime}-2}=1$. If we repeat the same argument, we can show that $x$ satisfies the following: $x_{2 i-1,2 i}=1$ for $i=1, \ldots k^{\prime}$ and $\sum_{\left(2 k^{\prime}+1, j\right) \in A^{\prime}} x_{2 k^{\prime}+1, j}=1$. But all odd nodes $j$ such that $j<2 k^{\prime}+1$ are assigned to some node, so node $2 k^{\prime}+1$ cannot be assigned to any of these nodes. Therefore such a point $x$ cannot be in $F_{Q}$.

Theorem 3. The k-triangle inequality (16) is facet defining for $P_{Q}$ if

1. $\{2 t-2,2 t-1,2 t+1\}$ is not a quadratic cover for all $t=2, \ldots, k$

2. $\{2 t, 2 m-1,2 m\}$ is not a quadratic cover for all $t=1, \ldots, k, m=1, \ldots, k$ such that $t \neq m$

3. $\{2 t, 2 m+1,2 m\}$ is not a quadratic cover for all $t=1, \ldots, k, m=1, \ldots, k$ such that $t \neq m$

4. $\{2 t+1,2 m+1,2 m\}$ is not a quadratic cover for all $t=1, \ldots, k, m=1, \ldots, k$ such that $t \neq m$

5. $\{2 t+1,2 m-1,2 m\}$ is not a quadratic cover for all $t=1, \ldots, k, m=1, \ldots, t-1$.

Proof: We prove that the k-triangle inequality (16) is facet defining for $P_{Q}$ by sequential lifting. For a given $k \geq 1$, define $O=\{(i, i+1): i=1,2, \ldots, 2 k\} \cup\{(2 k+1,1)\}$. The odd hole inequality $\sum_{(i, j) \in O} x_{i j} \leq k$ is facet defining for the polytope $P^{\prime}=\operatorname{conv}\left(F_{Q} \cap\{x \in\right.$ $\left.\{0,1\}^{n(n-1)}: x_{i j}=0 \forall(i, j) \in A \backslash O\right\}$ ) (see [19]). Let $L_{i j}$ be the set of variables that are lifted before $x_{i j}$. Define $F_{i j}=\left\{x \in F_{Q}: x_{t s}=0 \quad \forall(t, s) \in A \backslash\left(O \cup L_{i j} \cup\right.\right.$ $\left.\{(i, j)\}), x_{i j}=1\right\}$. Then $\sum_{(i, j) \in O} x_{i j}+\sum_{(i, j) \in A \backslash O} \pi_{i j} x_{i j} \leq k$ is facet defining for $P_{Q}$ where $\pi_{i j}=k-\max _{x \in F_{i j}}\left(\sum_{(t, s) \in O} x_{t s}+\sum_{(t, s) \in L_{i j}} \pi_{t s} x_{t s}\right)$ for all $(i, j) \in A \backslash O$.

We first lift variables $x_{i j}$ 's such that $(i, j) \in L=A^{\prime} \backslash O$. These are all $(i, j) \neq(2 k+1,1)$ such that $i \in I^{\prime}$ and $j \in I^{\prime}$ are odd and $i>j$. We do the lifting for $(i, j) \in L$ if all $(t, s) \in L$ such that $t<i$ or $t=i$ and $s>j$ are already lifted. We show that $\pi_{i j}=1$ for all $(i, j) \in L$ by induction on the order of the lifting variables. The first variable to lift is $x_{31}$. When $x_{31}=1, x_{12}=x_{23}=x_{34}=0$. The clique inequalities (11) imply that $x_{2 t, 2 t+1}+x_{2 t+1,2 t+2} \leq 1$ for all $2 \leq t \leq k-1$ and $x_{2 k, 2 k+1}+x_{2 k+1,1} \leq 1$. So $\sum_{(t, s) \in O} x_{t s} \leq k-1$ for all $x \in F_{31}$. As $x^{\prime}=e^{31}+\sum_{t=2}^{k} e^{2 t, 2 t+1}$ is in $F_{31}$ and $\sum_{(t, s) \in O} x_{t s}^{\prime}=k-1, \pi_{31}=1$.

We now lift $x_{i j}$ where $(i, j) \neq(3,1)$ and $\pi_{t s}=1$ for all $(t, s) \in L_{i j}$. As $x_{i j}=1$, we have that $x_{j, j+1}=0$. If node $j=2 k_{1}+1$, then the k-triangle inequality (16) for the first $2 k_{1}+1$ nodes imply that $\sum_{(t, s) \in A_{-}} x_{t s} \leq k_{1}$ where $A_{-}$is the set of arcs for the first $k_{1}$ triangles. So we get

$$
x_{2 k_{1}+1,2 k_{1}+2}+\sum_{(t, s) \in A_{-}} x_{t s} \leq k_{1} .
$$


As $x_{i j}=1$, we have that $x_{i, i+1}=0$. If node $i=2 k_{2}+1$, clique inequalities (11) imply that $x_{2 t, 2 t+1}+x_{2 t+1,2 t+2} \leq 1$ for all $k_{2}+1 \leq t \leq k-1$ and $x_{2 k, 2 k+1}+x_{2 k+1,1} \leq 1$. So

$$
x_{2 k_{2}+1,2 k_{2}+2}+\sum_{t=k_{2}+1}^{k-1}\left(x_{2 t, 2 t+1}+x_{2 t+1,2 t+2}\right)+x_{2 k, 2 k+1}+x_{2 k+1,1} \leq k-k_{2} .
$$

For $k_{1}+1 \leq v \leq k_{2}-1$ the clique inequalities (11) imply that

$$
x_{2 v, 2 v+1}+x_{2 v+1,2 v+2}+\sum_{(2 v+1, s) \in L_{i j}} x_{2 v+1, s} \leq 1 .
$$

If we sum up inequalities (17), (18) and (19) for $k_{1}+1 \leq v \leq k_{2}-1$, we obtain $\sum_{(t, s) \in O} x_{t s}+\sum_{(t, s) \in L_{i j}} x_{t s} \leq k_{1}+k-k_{2}+k_{2}-k_{1}-1=k-1$ for all $x \in F_{i j}$. The point $x^{\prime}=e^{i j}+\sum_{t=1, t \neq k_{2}}^{k} e^{2 t, 2 t+1}$ is in $F_{i j}$ and $\sum_{(t, s) \in O} x_{t s}^{\prime}+\sum_{(t, s) \in L_{i j}} x_{t s}^{\prime}=k-1$. So $\pi_{i j}=1$.

Next we show that $\pi_{i j}=0$ for all $(i, j) \in A \backslash A^{\prime}$. We first lift $x_{i j}$ such that $i$ and $j \neq i$ are not in $I^{\prime}$. As $x^{\prime}=e^{i j}+\sum_{t=1}^{k} e^{2 t, 2 t+1}$ is in $F_{i j}$ and $\sum_{(t, s) \in A^{\prime}} x_{t s}^{\prime}=k$, we conclude that $\pi_{i j}=0$.

For node $i \in I^{\prime}$ such that $i=2 k_{1}$, consider point $x^{\prime}=\sum_{t=1}^{k_{1}-1} e^{2 t-1,2 t}+\sum_{t=k_{1}+1}^{k} e^{2 t, 2 t+1}+$ $e^{2 k_{1}+1,2 k_{1}-1}+e^{i j}$ for some $j \notin I^{\prime}$. As $x^{\prime}$ is in $F_{i j}$ and $\sum_{(t, s) \in A^{\prime}} x_{t s}^{\prime}=k, \pi_{i j}=0$. We can also show that $\pi_{j i}=0$ in a similar way.

Consider $(i, j) \in A$ such that $i=2 k_{1}+1 \in I^{\prime}$ and $j \notin I^{\prime}$ or $j$ is an odd node in $I^{\prime}$ such that $i<j$ or $j$ is an even node in $I^{\prime}$ such that $i>j$. Define $x^{\prime}=\sum_{t=1}^{k_{1}} e^{2 t-1,2 t}+$ $\sum_{t=k_{1}+1}^{k} e^{2 t, 2 t+1}+e^{i j}$. Point $x^{\prime}$ is in $F_{i j}$ and $\sum_{(t, s) \in A^{\prime}} x_{t s}^{\prime}=k$. So $\pi_{i j}=0$. If $j \notin I^{\prime}$, we can show that $\pi_{j i}=0$ in a similar way.

If $(i, j) \in A$ such that both $i=2 k_{1}+1$ and $j=2 k_{2}$ are in $I^{\prime}, i<j$ and $j \neq i+1$, then consider $x^{\prime}=e^{i j}+\sum_{t=1}^{k_{1}} e^{2 t-1,2 t}+\sum_{t=k_{1}+1, t \neq k_{2}}^{k} e^{2 t, 2 t+1}+e^{2 k_{2}+1,2 k_{2}-1}$. Since $x^{\prime}$ is in $F_{i j}$ and $\sum_{(t, s) \in A^{\prime}} x_{t s}^{\prime}=k$, we have that $\pi_{i j}=0$.

The remaining variables are $x_{i j}$ 's such that $i$ is even and $j \neq i+1$. If $i=2 k_{1}$ and $j=2 k_{2}$ for some $k_{2} \leq k_{1}-1$ or $j=2 k_{2}+1$ for some $k_{2} \geq k_{1}+1$, then the point $x^{\prime}=e^{i j}+\sum_{t=1}^{k_{1}-1} e^{2 t-1,2 t}+\sum_{t=k_{1}+1}^{k} e^{2 t, 2 t+1}+e^{2 k_{1}+1,2 k_{1}-1}$ is in $F_{i j}$ and it satisfies $\sum_{(t, s) \in A^{\prime}} x_{t s}^{\prime}=k$. So $\pi_{i j}=0$.

If $i=2 k_{1}$ and $j=2 k_{2}$ for some $k_{2} \geq k_{1}+1$ or $j=2 k_{2}+1$ for some $k_{2} \leq k_{1}-1$, then $x^{\prime}=e^{i j}+\sum_{t=1}^{k_{1}-1} e^{2 t 1,2 t+1}+\sum_{t=k_{1}+1}^{k} e^{2 t-1,2 t}+e^{2 k+1,1}$ is in $F_{i j}$ and $\sum_{(t, s) \in A^{\prime}} x_{t s}^{\prime}=k$ showing that $\pi_{i j}=0$.

The proof of Theorem 3 shows that k-triangle inequalities are lifted odd hole inequalities. A 2-triangle in the conflict graph $G_{C}$ is given in Figure 4. The corresponding inequality is $x_{12}+x_{23}+x_{34}+x_{45}+x_{51}+x_{31}+x_{53} \leq 2$.

Clearly, there are lifted odd hole inequalities which are not k-triangle inequalities.

We now establish the complexity of the separation problem for k-triangle inequalities. The proof is given in the Appendix.

Theorem 4. The separation problem for the $k$-triangle inequalities is NP-complete. 


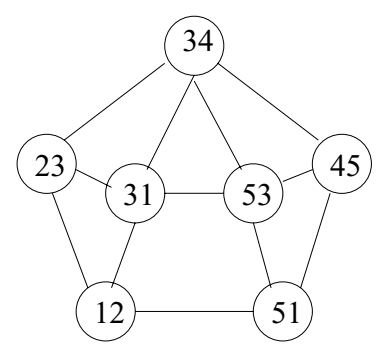

Figure 4. A 2-triangle structure in the conflict graph $G_{C}$

\subsection{3. $k$-leaf inequalities.}

Proposition 20. Given arc $(i, j) \in A$ and a subset $I^{\prime} \subseteq I \backslash\{i, j\}$ with $\left|I^{\prime}\right|=k$, the $k$-leaf inequality

$$
k x_{i j}+\sum_{t \in I \backslash\{i, j\}}(k-1) x_{i t}+\sum_{t \in I^{\prime}} x_{t i}+\sum_{t \in I^{\prime}} x_{j t} \leq k
$$

is valid for $P_{Q}$ for all $k \geq 1$.

Proof: For $x \in F_{Q}$, if $x_{i j}=1$, then as $x_{i t}=0$ for all $t \in I \backslash\{i, j\}, x_{j t}=0$ and $x_{t i}=0$ for all $t \in I^{\prime}$, inequality (20) is valid. If $x_{i s}=1$ for some $s \in I \backslash\{i, j\}$, then $x_{i t}=0$ for all $t \in I \backslash\{i, s\}$ and $x_{t i}=0$ for all $t \in I^{\prime}$. As $\sum_{t \in I^{\prime}} x_{j t} \leq 1$, inequality (20) is satisfied. If $\sum_{t \in I \backslash\{i\}} x_{i t}=0$, then as $x_{j t}+x_{t i} \leq 1$ for all $t \in I^{\prime}$, the k-leaf inequality is valid.

A k-leaf structure in the conflict graph $G_{C}$ is given in Figure 5. In this example, we have $I=\{1,2,3,4,5\}, i=1, j=2$ and $I^{\prime}=\{3,4\}$. The corresponding inequality is $2 x_{12}+x_{13}+x_{14}+x_{15}+x_{31}+x_{41}+x_{23}+x_{24} \leq 2$.

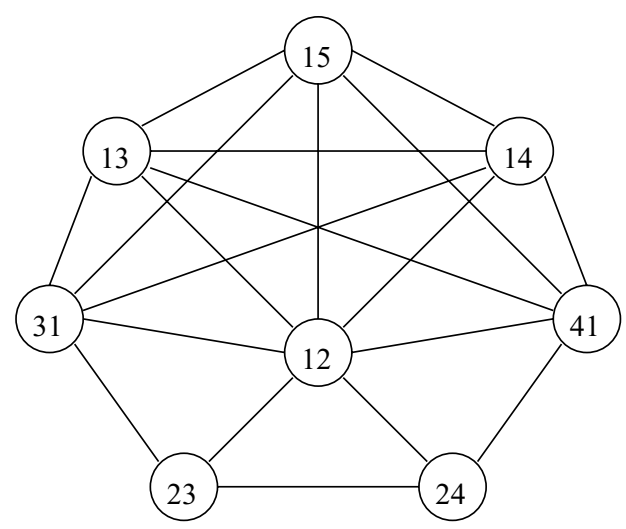

Figure 5. A k-leaf structure in the conflict graph $G_{C}$ 
Theorem 5. The k-leaf inequality (20) is facet defining for $P^{\prime}=\operatorname{conv}\left(F_{Q} \cap\{x \in\right.$ $\left.\left.\{0,1\}^{n(n-1)}: x_{l i}=0 \forall l \in I \backslash\left(I^{\prime} \cup\{i\}\right)\right\}\right)$ if and only if $I^{\prime} \cup\{i\}$ and $\{i, j, t\}$ for all $t \in I^{\prime}$ are not quadratic covers.

Proof: If $I^{\prime} \cup\{i\}$ is a quadratic cover, then cover inequality $\sum_{t \in I^{\prime}} x_{t i}+(k-1) \sum_{t \in I \backslash\{i\}} x_{i t} \leq$ $(k-1)$ is valid. If we sum this inequality and clique inequality (11) for $(i, j)$, we get $k x_{i j}+(k-1) \sum_{t \in I \backslash\{i, j\}} x_{i t}+\sum_{t \in I^{\prime}} x_{t i}+\sum_{t \in I \backslash\{j\}} x_{j t} \leq k$ which dominates the k-leaf inequality.

If there exists a node $l \in I^{\prime}$ such that $\{i, j, l\}$ is a quadratic cover, the inequality $x_{l j}+k x_{i j}+(k-1) \sum_{t \in I \backslash\{i, j\}} x_{i t}+\sum_{t \in I^{\prime}} x_{t i}+\sum_{t \in I^{\prime}} x_{j t} \leq k$ is the same as the k-leaf inequality if $x_{l j}=0$. If $x_{l j}=1$, then $x_{i l}+x_{l i}+\sum_{t \in I^{\prime}} x_{j t}=0$. As $\{i, j, l\}$ is a quadratic cover, we also have $x_{i j}=0$. So this inequality is valid. Moreover, it dominates the k-leaf inequality.

Assume that $I^{\prime} \cup\{i\}$ and $\{i, j, t\}$ for all $t \in I^{\prime}$ are not quadratic covers. Define $\bar{I}=$ $I \backslash\left(I^{\prime} \cup\{i\}\right)$, and $A_{0}=\{(l, i) \in A: l \in \bar{I}\}$. Define also $P_{f}=\left\{x \in P^{\prime}: k x_{i j}+\right.$ $\left.\sum_{t \in I \backslash\{i, j\}}(k-1) x_{i t}+\sum_{t \in I^{\prime}} x_{t i}+\sum_{t \in I^{\prime}} x_{j t}=k\right\}$. Assume that all points in $P_{f}$ satisfy $\sum_{(i, j) \in A \backslash A_{0}} \alpha_{i j} x_{i j}=\alpha_{0}$.

For $l \in I \backslash\{i, j\}$ and $m \in I \backslash\{i, j, l\}$, as $e^{i j}$ and $e^{i j}+e^{l m}$ are in $P_{f}, \alpha_{l m}=0$. For $l \in \bar{I} \backslash\{j\}$, as $x=\sum_{t \in I^{\prime}} e^{t i}$ and $x+e^{l j}$ are in $P_{f}, \alpha_{l j}=0$. Let $l \in I^{\prime}$. As both $e^{i j}$ and $e^{i j}+e^{l j}$ are in $P_{f}, \alpha_{l j}=0$. Now let $l \in \bar{I} \backslash\{j\}$. Both $x=\sum_{t \in I^{\prime}} e^{t i}$ and $x+e^{j l}$ are in $P_{f}$ proving that $\alpha_{j l}=0$.

Let $l \in I^{\prime}$. As both $x=\sum_{t \in I^{\prime}} e^{t i}$ and $x-e^{l i}+e^{j l}$ are in $P_{f}$, we have that $\alpha_{l i}=\alpha_{j l}=\alpha_{l}$. Since both $x$ and $e^{i j}$ are in $P_{f}, \alpha_{i j}=\sum_{t \in I^{\prime}} \alpha_{t}$.

Now, for $l \in I^{\prime}$, consider $x^{m}=e^{i l}+e^{j m}$ for all $m \in I^{\prime}$. As $e^{i j}$ and $x^{m}$ are all in $P_{f}$, we have that $\alpha_{i j}=\sum_{t \in I^{\prime}} \alpha_{t}=\alpha_{i l}+\alpha_{m}$ for all $m \in I^{\prime}$. This implies that $\alpha_{m}=\alpha$ for all $m \in I^{\prime}, \alpha_{i j}=k \alpha$ and $\alpha_{i l}=(k-1) \alpha$ for all $l \in I^{\prime}$.

For $l \in \bar{I} \backslash\{j\}$, as $e^{i j}$ and $e^{i l}+e^{j s}$ for some $s \in I^{\prime}$ are both in $P_{f}, k \alpha=\alpha_{i l}+\alpha$. So $\alpha_{i l}=(k-1) \alpha$ for all $l \in \bar{I} \backslash\{j\}$. If we plug in $e^{i j}$ we can show that $\alpha_{0}=k \alpha$.

Define for $j \in I, I_{0} \subseteq I$ and $I_{1} \subseteq I$

$$
\begin{aligned}
\operatorname{Cap}_{j}\left(I_{1}, I_{0}\right)= & \sum_{i \in I \backslash\left(I_{0} \cup I_{1}\right)}\left(a_{i}+\sum_{m \in I_{0}} T_{i m}-\sum_{m \in I_{1}} T_{i m}\right) x_{i j} \\
& +\sum_{i \in I_{1}}\left(a_{i}+\sum_{m \in I \backslash I_{1}} T_{i m}\right)+\sum_{i \in I \backslash\left(I_{0} \cup I_{1}\right)} \sum_{m \in I \backslash\left(I_{0} \cup I_{1}\right)} T_{i m} x_{i j}\left(1-x_{m j}\right) .
\end{aligned}
$$

The value $\operatorname{Cap}_{j}\left(I_{1}, I_{0}\right)$ is the left hand side of the capacity constraint for node $j$ when nodes in $I_{1}$ are assigned to node $j$ and the nodes in $I_{0}$ are assigned to some other concentrators. In this case, the capacity constraint is equivalent to $\operatorname{Cap}_{j}\left(I_{1}, I_{0}\right) \leq M$. 
Now we lift the variables whose values are fixed to 0 . Let $\pi_{l}$ denote the optimal lifting coefficient for $x_{l i}$ with $l \in \bar{I}$ in

$$
k x_{i j}+\sum_{t \in I \backslash\{i, j\}}(k-1) x_{i t}+\sum_{t \in I^{\prime}} x_{t i}+\sum_{t \in I^{\prime}} x_{j t}+\sum_{l \in \bar{I}} \pi_{l} x_{l i} \leq k
$$

Proposition 21. Define $I^{0}=\left\{l \in \bar{I} \backslash\{j\}: \exists t_{l} \in I^{\prime}:\left(I^{\prime} \backslash\left\{t_{l}\right\}\right) \cup\{i, l\}\right.$ is not a quadratic cover $\}$ and $I^{+}=\bar{I} \backslash\left(I^{0} \cup\{j\}\right)$. Suppose that we first lift variables $x_{l i}$ 's such that $l \in I^{0}$, then $x_{j i}$ and then variables $x_{l i}$ 's such that $l \in I^{+}$.

1. $\pi_{l}=0$ for all $l \in I^{0}$.

2. $\pi_{j}=\min _{C \in \bar{C}}|C|$ where $\bar{C}=\left\{C \subseteq I^{\prime}:\left(I^{\prime} \backslash C\right) \cup\{i, j\}\right.$ is not a quadratic cover $\}$.

3. For $l \in I^{+}$, define $L_{l} \subset I^{+}$to be the set of indices of variables that are lifted before $x_{l i}$. The lifting coefficient of $x_{l i}$ is $\pi_{l}=k-\max \left\{\pi_{l}^{0}, \pi_{l}^{1}\right\}$ where

$$
\begin{aligned}
\pi_{l}^{0}=\pi_{j}+\max & \sum_{t \in I^{\prime}} x_{t i}+\sum_{t \in L_{l}} \pi_{t} x_{t i} \\
\text { s.t. } & \operatorname{Cap}_{i}\left(\{i, j, l\}, I^{0} \cup\left(I^{+} \backslash\left(L_{l} \cup\{l\}\right)\right)\right) \leq M \\
& x_{t i} \in\{0,1\} \quad \forall t \in I^{\prime} \cup L_{l}
\end{aligned}
$$

and

$$
\begin{aligned}
\pi_{l}^{1}=1+\max & \sum_{t \in I^{\prime}} x_{t i}+\sum_{t \in L_{l}} \pi_{t} x_{t i} \\
\text { s.t. } & \operatorname{Cap}_{i}\left(\{i, l\}, I^{0} \cup\left(I^{+} \backslash\left(L_{l} \cup\{l\}\right)\right) \cup\{j\}\right) \leq M \\
& x_{t i} \in\{0,1\} \quad \forall t \in I^{\prime} \cup L_{l} .
\end{aligned}
$$

Proof: For $l \in I^{0}$, let $t_{l} \in I^{\prime}$ be such that $\left(I^{\prime} \backslash\left\{t_{l}\right\}\right) \cup\{i, l\}$ is not a quadratic cover. As $x=e^{l i}+\sum_{t \in I^{\prime} \backslash\left\{t_{l}\right\}} e^{t i}+e^{j t_{l}}$ is in $F_{Q}, x_{l i}=1$ and $\sum_{t \in I^{\prime}}\left(x_{t i}+x_{j t}\right)=k, \pi_{l}=0$ for $l \in I^{0}$. Now we prove the second statement. The value $\pi_{j}$ is

$$
\begin{gathered}
\pi_{j}=k-\max \sum_{t \in I^{\prime}} x_{t i}=\min \sum_{t \in I^{\prime}}\left(1-x_{t i}\right) \\
\text { s.t. } \operatorname{Cap}_{i}\left(\{i, j\}, I \backslash\left(I^{\prime} \cup\{i, j\}\right)\right) \leq M \\
\qquad x_{t i} \in\{0,1\} \quad \forall t \in I^{\prime} .
\end{gathered}
$$

So $\pi_{j}$ is the minimum number of nodes that should be removed from the set $I^{\prime}$ so that the remaining nodes can be assigned to node $i$ with node $j$. 


\section{LABBÉ AND YAMAN}

We now prove the third statement. For $x_{l i}$ with $l \in I^{+}, \pi_{l}$ is

$$
\begin{aligned}
\pi_{l}=k-\max & \sum_{t \in I^{\prime}} x_{t i}+\sum_{t \in L_{l}} \pi_{t} x_{t i}+\sum_{t \in I^{\prime}} x_{j t}+\pi_{j} x_{j i} \\
\text { s.t. } & \operatorname{Cap}_{i}\left(\{i, l\}, I^{0} \cup\left(I^{+} \backslash\left(L_{l} \cup\{l\}\right)\right)\right) \leq M \\
& x_{j i}+\sum_{t \in I^{\prime}} x_{j t} \leq 1 \\
& x_{j t}+x_{t i} \leq 1 \quad \forall t \in I^{\prime} \cup L_{l} \\
& x_{t i} \in\{0,1\} \quad \forall t \in I^{\prime} \cup L_{l} \cup\{j\} \\
x_{j t} & \in\{0,1\} \quad \forall t \in I^{\prime} .
\end{aligned}
$$

We investigate two cases:

1. If $x_{j i}=1, \pi_{l} \leq k-\pi_{l}^{0}$.

2. If $x_{j i}=0$, then as there is at least on node in $I^{\prime}$ that cannot be assigned to node $i$ when the other nodes in $I^{\prime}$ and node $l$ are assigned to node $i$. Node $j$ can be assigned to this node. So $\pi_{l} \leq k-\pi_{l}^{1}$.

If $x_{j i}$ is lifted after $x_{l i}$ for $l \in I^{+}$, then $\pi_{l}=k-\pi_{l}^{1}$ and $\pi_{j}=k-\pi_{j}^{1}+1$.

Proposition 22. The separation problem for k-leaf inequalities can be solved in $O\left(n^{3}\right)$ time.

Proof: For a fractional $x^{*},(i, j) \in A$ and $I^{\prime} \subseteq I \backslash\{i, j\}$, define $v\left(I^{\prime},(i, j)\right)=-x_{i}^{*}+$ $\sum_{t \in I^{\prime}}\left(x_{i j}^{*}+x_{i}^{*}+x_{t i}^{*}+x_{j t}^{*}-1\right)$ where $x_{i}^{*}=\sum_{t \in I \backslash\{i, j\}} x_{i t}^{*}$. The separation problem is equivalent to maximizing $v\left(I^{\prime},(i, j)\right)$. For $(i, j) \in A, I^{*}=\left\{t \in I \backslash\{i, j\}: x_{i j}^{*}+x_{i}^{*}+x_{t i}^{*}+x_{j t}^{*}-1>0\right\}$ is a maximizing set.

\subsubsection{2-cycle inequalities.}

Proposition 23. Let $D \subset I$ with $|D|=3$ and $c \in I \backslash D$. Let $C$ be a directed cycle on the nodes of $D$. Renumber the nodes such that $D=\{1,2,3\}$, the cycle is $1,2,3,1$, the node $c=4$ and $I \backslash D=\{5,6, \ldots, n\}$.

1. The 2-cycle inequality

$$
2 x_{12}+2 x_{23}+2 x_{31}+x_{14}+x_{24}+x_{34}+\sum_{i \in I \backslash\{4\}} x_{4 i} \leq 3
$$

is valid for $P_{Q}$. 


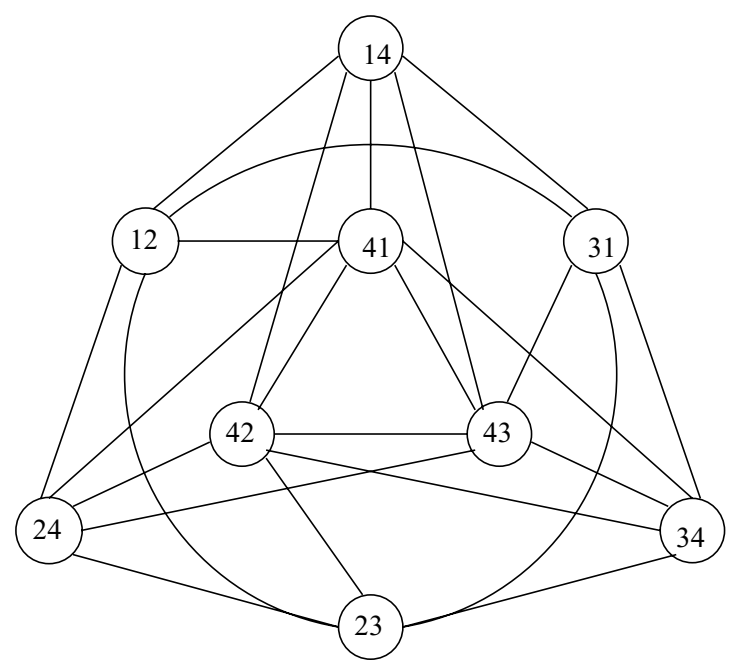

Figure 6. A 2-cycle structure in the conflict graph $G_{C}$

2. Choose a node $m \in D$ and let $m^{\prime}$ be the node in $D$ such that $\left(m^{\prime}, m\right) \in C$. Renumber the nodes such that $m=1$ and $m^{\prime}=3$. Then the 2-cycle inequality

$$
2 x_{12}+2 x_{23}+2 x_{31}+x_{14}+x_{24}+x_{34}+x_{41}+x_{42}+x_{13}+\sum_{i=5}^{n} x_{1 i} \leq 3
$$

is valid for $P_{Q}$.

Proof: We prove the first statement. The second one can be proved in a similar way. Consider the following inequalities: $x_{12}+x_{23}+x_{31} \leq 1, x_{12}+x_{14}+x_{31} \leq 1, x_{23}+x_{24}+x_{12} \leq$ $1, x_{31}+x_{34}+x_{23} \leq 1, \sum_{i \in I \backslash\{4\}} x_{4 i}+x_{34} \leq 1$ and $x_{12}+x_{23}+x_{31}+x_{14}+x_{24}+\sum_{i \in I \backslash\{4\}} x_{4 i} \leq 2$, where the first inequality is a triangle inequality, the following four inequalities are implied by the clique inequalities and the last one is a $W-2$ inequality where $W=\{1,2,3,4\}$ and $U=\{4\}$. If we sum up these inequalities, divide by 2 and round down the right hand side, we get inequality (23).

A 2-cycle structure in the conflict graph $G_{C}$ is given in Figure 6. The corresponding inequality is $2 x_{12}+2 x_{23}+2 x_{31}+x_{14}+x_{24}+x_{34}+\sum_{i=1}^{3} x_{4 i} \leq 3$.

Theorem 6. If $\{1, \ldots, 4\}$ is not a quadratic cover and $I^{\prime} \cup\{i\}$ is not a quadratic cover for all $I^{\prime} \subset\{1, \ldots, 4\}$ such that $\left|I^{\prime}\right|=2$ and for all $i \in I \backslash\{1 \ldots, 4\}$, then the 2-cycle inequalities (23) and (24) define facets of $P_{Q}$.

Proof: The PORTA [8] output given in [21] for $n=4$ shows that both inequalities are facet defining for $P_{U}$ when $n=4$. As $\{1, \ldots, 4\}$ is not a quadratic cover, $P_{Q}=P_{U}$. We 
prove that inequality (23) is facet defining for $P_{Q}$ for any $n>4$ by lifting. The proof for (24) can be done in a similar way.

Consider the polytope $P^{\prime}=\operatorname{conv}\left(F_{Q} \cap\left\{x \in\{0,1\}^{n(n-1)}: x_{i j}=0\right.\right.$ if $i>4$ or $\left.\left.j>4\right\}\right)$. Inequality (23) is facet defining for $P^{\prime}$. Let $L$ be the set of indices of variables fixed to 0 and $L_{i j}$ be the set of variables that are lifted before $x_{i j}$ with $(i, j) \in L$. Denote by $\pi_{i j}$ the optimal lifting coefficient of $x_{i j}$. Define $F_{i j}=\left\{x \in F_{Q}: x_{t s}=0 \quad \forall(t, s) \in\right.$ $\left.L \backslash\left(L_{i j} \cup\{(i, j)\}\right), x_{i j}=1\right\}$. Then $\pi_{i j}=3-\max _{x \in F_{i j}}\left(2\left(x_{12}+x_{23}+x_{31}\right)+x_{14}+x_{24}+\right.$ $\left.x_{34}+\sum_{i \in I \backslash\{4\}} x_{4 i}+\sum_{(t, s) \in L_{i j}} \pi_{t s} x_{t s}\right)$. Suppose, we first lift $x_{i j}$ such that $i>4$ and $j>4$. As $e^{12}+e^{42}+e^{i j}$ is in $F_{i j}$, we have $\pi_{i j}=0$. Now consider $x_{i j}$ such that $i>4$ and $j \leq 3$. Define $x=e^{l m}+e^{4 j}+e^{i j}$ where $l \neq j$ and $m \neq j$ and $(l, m) \in C$. The point $x$ is in $F_{i j}$, so $\pi_{i j}=0$. If we are lifting $x_{i 4}$ with $i>4$, then as $e^{12}+e^{34}+e^{i 4}$ is in $F_{i 4}$, $\pi_{i 4}=0$. Consider $x_{i j}$ such that $i \leq 3$ and $j \geq 5$. As $e^{k l}+e^{4 l}+e^{i j}$ where $(k, l) \in C$ and $k, l \neq i$ is in $F_{i j}, \pi_{i j}=0$. The only remaining variables are $x_{4 i}$ 's where $i \geq 5$. Suppose that $x_{4 i}=1$. Then $x_{14}=x_{24}=x_{34}=\sum_{(4, j) \in L_{4 i}} x_{4 j}=0$. The lifting coefficient $\pi_{4 i}=3-\max _{x \in F_{4 i}}\left(2 x_{12}+2 x_{23}+2 x_{31}\right)$. Triangle inequality for $\{1,2,3\}$ implies that $2 x_{12}+2 x_{23}+2 x_{31} \leq 2$ for any $x \in F_{4 i}$. As $e^{12}+e^{4 i}$ is in $F_{4 i}$, we have that $\pi_{4 i}=1$.

\subsection{Binpacking inequalities}

The capacitated facility location problem with single assignment (CFLPS) is defined on two sets $I$ and $J$, the set of terminals and the set of possible locations for concentrators, respectively. Each terminal node should be assigned to exactly one location where a concentrator is installed. Deng and Simchi-Levi [9] introduce binpacking inequalities for the polytope of CFLPS.

Let $I^{\prime} \subseteq I$ and $J^{\prime} \subseteq I$ and $b\left(I^{\prime}\right)$ be the minimum number of concentrators to be installed to assign all nodes in $I^{\prime}$. The binpacking inequality

$$
\sum_{i \in I^{\prime}} \sum_{j \in J^{\prime} \backslash\{i\}} x_{i j}-\sum_{j \in J^{\prime} \backslash I^{\prime}}\left(1-\sum_{m \in I \backslash\{j\}} x_{j m}\right) \leq\left|I^{\prime}\right|-b\left(I^{\prime}\right)
$$

is valid for $P_{Q}$.

The computation of $b\left(I^{\prime}\right)$ does not take into account that a node which receives a concentrator is assigned to itself.

Define $F_{B P}=\left\{x \in\{0,1\}^{\left|I^{\prime}\right|^{2}}: \sum_{j \in I^{\prime}} x_{i j}=1 \forall i \in I^{\prime}, \sum_{i \in I^{\prime}} a_{i} x_{i j} \leq M x_{j j} \forall j \in I^{\prime}\right\}$ and $\bar{b}\left(I^{\prime}\right)=\min _{x \in F_{B P}} \sum_{j \in I^{\prime}} x_{j j}$.

Proposition 24. Let $I^{\prime} \subseteq I$. The modified binpacking inequality

$$
\sum_{i \in I^{\prime}} \sum_{j \in I^{\prime} \backslash\{i\}} x_{i j} \leq\left|I^{\prime}\right|-\bar{b}\left(I^{\prime}\right)
$$

is valid for $P_{Q}$. 
Proof: Let $x \in F_{Q}$ and $J=\left\{j \in I \backslash I^{\prime}: \sum_{i \in I^{\prime}} x_{i j} \geq 1\right\}$. Since all nodes in $I^{\prime} \cup J$ are assigned to nodes in the same set, we have $\sum_{i \in I^{\prime} \cup J} \sum_{j \in I^{\prime} \cup J \backslash\{i\}} x_{i j} \leq\left|I^{\prime}\right|+|J|-\bar{b}\left(I^{\prime} \cup J\right)$. Since all nodes in $J$ are concentrator nodes, they are not assigned to any other node, i.e., $\sum_{i \in J} \sum_{j \in I^{\prime} \cup J \backslash\{i\}} x_{i j}=0$. Hence $\sum_{i \in I^{\prime}} \sum_{j \in I^{\prime} \cup J \backslash\{i\}} x_{i j} \leq\left|I^{\prime}\right|+|J|-\bar{b}\left(I^{\prime} \cup J\right)$. As $\sum_{i \in I^{\prime}} \sum_{j \in J} x_{i j} \geq|J|$, we obtain $\sum_{i \in I^{\prime}} \sum_{j \in I^{\prime} \backslash\{i\}} x_{i j} \leq\left|I^{\prime}\right|-\bar{b}\left(I^{\prime} \cup J\right)$. Now since, $\bar{b}\left(I^{\prime} \cup J\right) \geq \bar{b}\left(I^{\prime}\right)$, inequality (25) is satisfied.

Theorem 7. Let $I^{\prime} \subseteq I$ be such that $\bar{b}\left(I^{\prime} \backslash\{i\}\right)=\bar{b}\left(I^{\prime}\right)-1$ for all $i \in I^{\prime}$. Then the modified binpacking inequality (25) is facet defining for $P_{L}$.

Proof: Assume, without loss of generality, that $I^{\prime}=\left\{1,2, \ldots n^{\prime}\right\}$ and $a_{1} \leq a_{2} \leq \cdots \leq$ $a_{n^{\prime}}$. Define $P_{f}=\left\{x \in P_{L}: \sum_{i \in I^{\prime}} \sum_{j \in I^{\prime} \backslash\{i\}} x_{i j}=\left|I^{\prime}\right|-\bar{b}\left(I^{\prime}\right)\right\}$. Assume also that $\bar{b}\left(I^{\prime} \backslash\{i\}\right)=$ $\bar{b}\left(I^{\prime}\right)-1$ for all $i \in I^{\prime}$ and that all $x$ in $P_{f}$ satisfy $\sum_{i \in I} \sum_{j \in I \backslash\{i\}} \alpha_{i j} x_{i j} \leq \alpha_{0}$.

Consider a solution where node $i$ is free, $\bar{b}\left(I^{\prime}\right)-1$ nodes are open so that the remaining nodes are assigned to these nodes. Such a solution satisfies the modified binpacking inequality (25) at equality. Let $x^{i}$ denote such a solution. Choose two nodes $k$ and $l$ not in $I^{\prime}$. As both $x^{i}$ and $x^{i}+e^{k l}$ are in $P_{f}$, we have $\alpha_{k l}=0$. Similarly we can show that $\alpha_{l i}=\alpha_{i l}=0$ since node $i$ is free in $x$. For each $l \in I^{\prime} \backslash\{i\}$, there exists an $x^{i}$ where node $l$ is assigned to some node. As both $x^{i}+e^{l i}$ and $x^{i}+e^{i l}$ are in $P_{f}$, we have $\alpha_{l i}=\alpha_{i l}$ for all $i \in I^{\prime}$ and $l \in I^{\prime} \backslash\{i\}$.

Now take some $x^{1}$ and let $I_{t}$ denote the set of the $t$ th open node and the nodes assigned to it. As $\bar{b}\left(I^{\prime} \backslash\{1\}\right)=\bar{b}\left(I^{\prime}\right)-1$ and as any two nodes can be assigned together, each $I_{t}$ has at least two nodes. Assume that $l$ is the open node in $I_{t}$. Let $j$ be another node in $I_{t}$ which is assigned to $l$. Replace node $j$ by node 1 . This is feasible since $a_{1} \leq a_{j}$. As the resulting point is in $P_{f}$, we have $\alpha_{1 l}=\alpha_{j l}=\alpha_{l}$ for all $j \in I_{t} \backslash\{l\}$. As any node in $I_{t}$ can be the open node, we have that $\alpha_{j}\left(\left|I_{t}\right|-1\right)=\alpha_{l}\left(\left|I_{t}\right|-1\right)$ for any two nodes $j$ and $l$ in $I_{t}$. This shows that $\alpha_{j}=\alpha^{t}$ if $j \in I_{t}$. Repeating the same argument for nodes $2,3 \ldots, n^{\prime}$ we can show that $\alpha_{i j}=\alpha^{t}$ if $i<j$ and $j \in I_{t}$. As $\alpha_{i j}=\alpha_{j i}$, we conclude that $\alpha_{i j}=\alpha^{t}$ if $\max \{i, j\} \in I_{t}$.

Consider some $x^{1}$. Choose $I_{m}$ and $I_{l}$. Let $s_{m}$ and $s_{l}$ denote the smallest nodes in $I_{m}$ and $I_{l}$ respectively. Assume, without loss of generality, that nodes $s_{m}$ and $s_{l}$ are not open in $x^{1}$ and that $s_{m}<s_{l}$. Define $x$ to be the same as $x^{1}$ except that node $s_{l}$ is free, all nodes in $I_{l} \backslash\left\{s_{l}\right\}$ are assigned to node $s_{m}$ and node 1 is assigned to the open node in $I_{m}$. Figure 7 depicts how we construct $x$ starting from $x^{1}$. Define also $x^{\prime}$ to be the same as $x$ except that node 1 is assigned to node $s_{l}$. Both $x$ and $x^{\prime}$ are in $P_{f}$. So $\left(\left|I_{l}\right|-1\right) \alpha^{l}+\left(\left|I_{m}\right|-1\right) \alpha^{m}=$ $\left(\left|I_{l}\right|-1\right) \alpha^{l}+\left(\left|I_{m}\right|-2\right) \alpha^{m}+\alpha^{l}$. This shows that $\alpha^{m}=\alpha^{l}$. Therefore $\alpha_{i j}=\alpha$ for all $i \in I^{\prime}$ and $j \in I^{\prime} \backslash\{i\}$. As in $x^{1}, \bar{b}\left(I^{\prime}\right)-1$ nodes are open, node 1 is free and the remaining nodes are assigned to these open nodes, $\alpha_{0}=\alpha\left(I^{\prime}-1-\bar{b}\left(I^{\prime}\right)+1\right)=\alpha\left(I^{\prime}-\bar{b}\left(I^{\prime}\right)\right)$. $P_{Q}$.

We now present a family of binpacking inequalities some of which are facet defining for

Proposition 25. Let $I^{\prime} \subseteq I$. If $I_{k}$ is a quadratic cover for all $I_{k} \subseteq I^{\prime}$ with $\left|I_{k}\right|=k$, then 


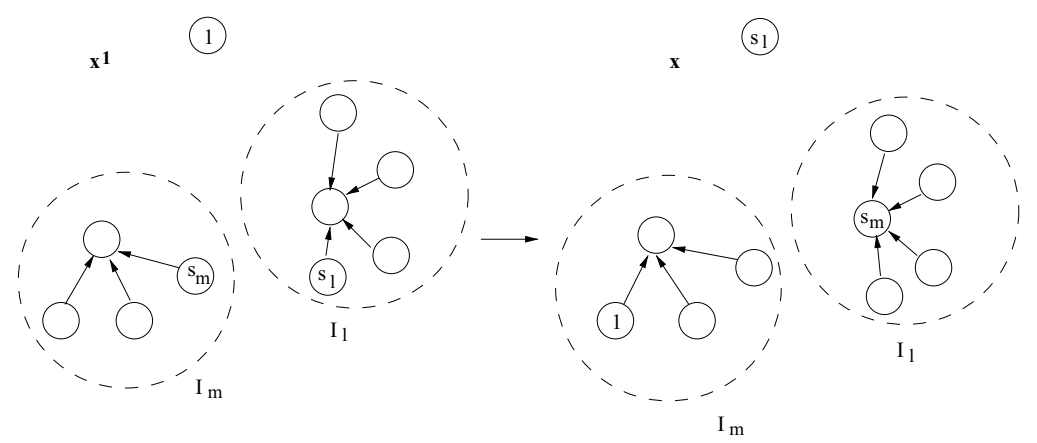

Figure 7. From $x^{1}$ to $x$

the quadratic binpacking inequality

$$
\sum_{i \in I^{\prime}} \sum_{j \in I^{\prime} \backslash\{i\}} x_{i j} \leq\left\lfloor\frac{\left|I^{\prime}\right|(k-2)}{k-1}\right\rfloor
$$

is valid for $P_{Q}$.

Proof: The quadratic cover inequality $\sum_{j \in I^{\prime} \backslash\{i\}}(k-2) x_{i j}+\sum_{j \in I^{\prime} \backslash\{i\}} x_{j i} \leq k-2$ is valid for $i \in I^{\prime}$. If we sum these inequalities over all $i \in I^{\prime}$, divide by $k-1$ and round down the right hand side, we obtain inequality (26).

Theorem 8. Let $I^{\prime} \subseteq I$. Define $a=\left\lfloor\frac{\left|I^{\prime}\right|}{k-1}\right\rfloor$ and $r=\left|I^{\prime}\right|-a(k-1)$. If $I_{k}$ is a quadratic cover for all $I_{k} \subseteq I^{\prime}$ with $\left|I_{k}\right|=k, I_{k-1}$ is not a quadratic cover for all $I_{k-1} \subseteq I^{\prime}$ with $\left|I_{k-1}\right|=k-1$ and $r=1$, then the quadratic binpacking inequality (26) is facet defining for $P_{Q}$.

Proof: Assume that $I_{k}$ is a quadratic cover for all $I_{k} \subseteq I^{\prime}$ with $\left|I_{k}\right|=k, I_{k-1}$ is not a quadratic cover for all $I_{k-1} \subseteq I^{\prime}$ with $\left|I_{k-1}\right|=k-1$ and $r=1$. Inequality (26) becomes $\sum_{i \in I^{\prime}} \sum_{j \in I^{\prime} \backslash\{i\}} x_{i j} \leq a(k-2)$. Define $P_{f}=\left\{x \in P_{Q}: \sum_{i \in I^{\prime}} \sum_{j \in I^{\prime} \backslash\{i\}} x_{i j}=a(k-2)\right\}$. Assume that all $x \in P_{f}$ satisfy $\sum_{(i, j) \in A} \alpha_{i j} x_{i j}=\alpha_{0}$.

Let $l \in I^{\prime}$. Choose a subset $I_{o}^{\prime} \in I^{\prime} \backslash\{l\}$ with $\left|I_{o}^{\prime}\right|=a$ to be the set of open nodes. We partition the set $I^{\prime} \backslash\left(I_{o}^{\prime} \cup\{l\}\right)$ into sets $I_{1}^{\prime}, \ldots, I_{a}^{\prime}$ where $\left|I_{t}^{\prime}\right|=k-2$ for all $t=1, \ldots, a$. Assign the nodes in $I_{t}^{\prime}$ to the $t$ th open node. Such a solution $x$ is in $P_{f}$. Moreover, node $l$ is free in $x$. Let $X_{l}$ be the set of such solutions.

Take two nodes $i$ and $j \neq i$ that are not in $I^{\prime}$ and $x \in X_{l}$. As $x+e^{i j}$ is also in $P_{f}$, we have that $\alpha_{i j}=0$ for all $i \in I \backslash I^{\prime}$ and $j \in I \backslash\left(I^{\prime} \cup\{i\}\right)$.

Consider $l \in I^{\prime}$ and $x \in X_{l}$. Take also a node $m \in I \backslash I^{\prime}$. Both $x+e^{m l}$ and $x+e^{l m}$ are in $P_{f}$. So $\alpha_{m l}=\alpha_{l m}=0$ for all $l \in I^{\prime}$ and $m \in I \backslash I^{\prime}$.

Let $l \in I^{\prime}$ and $(m, j) \in A$ be such that both $m$ and $j$ are in $I^{\prime} \backslash\{l\}$. As any $k-1$ nodes can be assigned together, there exists a solution $x \in X_{l}$ such that $x_{m j}=1$. As $x-e^{m j}+e^{l j}$ 
is also in $P_{f}, \alpha_{l j}=\alpha_{m j}$. As we can repeat the same argument for other nodes $m \in I^{\prime} \backslash\{l\}$, we can show that $\alpha_{l j}=\alpha_{j}$ for all $l \in I^{\prime} \backslash\{j\}$.

Consider nodes $l$ and $m$ in $I^{\prime}$ and $x \in X_{l}$ where node $m$ is open. Define $x^{\prime}$ to be the same as $x$ except that we change node $m$ by node $l$. We have that $(k-2) \alpha_{l}=(k-2) \alpha_{m}$. So $\alpha_{l}=\alpha$ for all $l \in I^{\prime}$. Further, $\alpha_{0}=\alpha a(k-2)$.

\section{The branch and cut algorithm}

In this section, we present the branch and cut algorithm and the computational results. The algorithm is is developed and tested under the Linux operating system distribution of SuSe 7.2. It is implemented in C++ using ABACUS 2.3 (see [14]) and the LP solver CPLEX 7.0. The runs are taken on an Intel Pentium III, $1 \mathrm{GHz}$ and 1 GB RAM.

To linearize the formulation of the QCL, we replace the quadratic capacity constraints (4) by

$$
\sum_{i \in I} a_{i} x_{i j}+\sum_{(i, m) \in K^{\prime}} T_{i m}\left(x_{i j}-x_{m j}\right) \leq M x_{j j} \quad \forall K^{\prime} \subseteq K, j \in I .
$$

As the number of such constraints grows exponentially with $n$, they are not included in the formulation. We add them when they are violated.

We start with the LP relaxation that contains constraints (2) and $x_{i j} \geq 0$ for all $i, j \in I$. We run a preprocessing algorithm which is a simplification of the procedure given in [15]. This procedure first identifies quadratic covers of size one and two. If there is a cover of size one, then the problem is infeasible since the corresponding terminal cannot be assigned to any node. If a node is not a cover but it forms a cover with any other node, then this node is assigned to itself and is removed from the problem. For covers of size two, say $i$ and $j \neq i$, the algorithm adds cover inequalities $x_{i m}+x_{j m} \leq x_{m m}$ for all $m \in I \backslash\{i, j\}$ and removes the variables $x_{i j}$ and $x_{j i}$ (they are 0 in any feasible solution). It adds inequalities (3) which are not dominated by the cover inequalities.

During preprocessing, we compute lower bounds on the quadratic terms that appear in the capacity constraints (using the procedure Compute Traffic given in [15]). Using these lower bounds we obtain linear relaxations of the quadratic capacity constraints. Then we compute the $L_{2}$ bound of [17] for the resulting bin packing structure. This bound is a lower bound on the number of concentrators to be installed. We add the corresponding inequality to the formulation. The reader can refer to [15] for details of the preprocessing procedure.

The solution $x^{*}$ of the current LP relaxation is feasible if $x^{*}$ is integer and if it satisfies inequalities (27). If $x^{*}$ is not integer, we apply the rounding heuristic given in [15]. Let $x^{\prime}$ be the solution of the heuristic. For $i \in I$, if $x_{i i}^{*} \geq 0.5$, then node $i$ is assigned to itself, i.e., $x_{i i}^{\prime}=1$. Otherwise it is assigned to some node $j \in \operatorname{argmax}_{l \in I} x_{i l}^{*}$. If there exists $i$ and $j$ such that $x_{i j}^{\prime}=1$ but $x_{j j}^{\prime}=0$, then node $j$ is assigned to itself. If the current solution $x^{\prime}$ satisfies the capacity constraints then the heuristic stops. If not, it iterates at most 15 times as follows. The slack capacities are computed. Let $s_{1}$ be the minimum slack and $s_{n}$ be the maximum slack. If the sum of slack capacities is nonnegative and there exists a node $i$ assigned to the node with minimum slack and $s_{n}>a_{i} \geq-s_{1}$, then node $i$ is 
assigned to the node with maximum slack. If these conditions are not satisfied then a node $j^{*} \in \operatorname{argmax}_{l \in I: x_{l l}^{\prime}=0} \sum_{i \in I} x_{i l}^{*}$ is assigned to itself.

We branch on the most fractional $x_{j j}$ variable, i.e., we branch on $x_{j j}$ if $j \in \operatorname{argmin}_{i \in I} \mid x_{i i}^{*}$ $-0.5 \mid$. If all $x_{j j}^{*}$ 's are integer then we branch on the most fractional variable $x_{i j}$. We expect to have a more balanced tree by branching on the variables $x_{j j}$ 's first.

The enumeration strategy is the best first strategy.

To face tailing-off, we resort to branching if the improvement over the five consecutive LP's is less than $0.05 \%$.

\subsection{Separation algorithms}

Cover inequalities: Initial tests showed that separation and lifting for quadratic cover inequalities are too expensive. So we separate the cover inequalities on linear knapsack inequalities (27). As for a given $j \in I$, the problem of finding the set $K^{\prime}$ which defines a knapsack inequality (27) for which there is a violated cover inequality is the same problem as the separation of quadratic cover inequalities, we use the following heuristic: we take $K^{\prime}=\left\{(i, m) \in K: x_{i j}^{*}-x_{m j}^{*}>0\right\}$. The separation and lifting are done as explained in [15]. For $j \in I$, let $I_{1}=\left\{i \in I: x_{i j}^{*}=x_{j j}^{*}\right\}$ and $I_{0}=\left\{i \in I: x_{i j}^{*}=0\right\}$. If the inequality corresponding to cover $C$ is violated when $x_{i j}=1$ for all $i \in I_{1}$ and $x_{i j}=0$ for all $i \in I_{0}$, then $x_{i j}$ for $i \in I \backslash\left(I_{1} \cup I_{0} \cup C\right)$ is fixed to 0 . The lifting order is as follows: variables $x_{i j}$ whose values are fixed to 0 and who have $x_{i j}^{*}>0$, variables whose values are fixed to 1 except $x_{j j}$, the remaining variables whose values are fixed to 0 and finally $x_{j j}$. This is similar to the order given by [12].

Quadratic binpacking inequalities on three nodes and(1-)triangle inequalities are separated by enumeration. If nodes $\{i, j, l\}$ form a quadratic cover, then we check whether the quadratic binpacking inequality is violated. If these nodes do not form a quadratic cover, then we check if one of the triangle inequalities is violated.

$W$-2 inequalities with $|W| \geq 4$ are separated using the algorithm given in [3].

$k$-triangle inequalities with $k \geq 2$ : As the separation problem for the $\mathrm{k}$-triangle inequalities is NP-complete, we use the greedy heuristic given in [15]. Each ordered triple $(i, j, l)$ such that $x_{i j}^{*}>0$ and $x_{i j}^{*}+x_{j l}^{*}+x_{l i}^{*}>0.8$ is considered as a first triangle. Iteratively, two new nodes which have a contribution of at least 0.8 to the left hand side are appended as a triangle until a violated k-triangle inequality with $k \geq 2$ is found.

$k$-leaf inequalities with $k \geq 2$ are separated exactly in $O\left(n^{3}\right)$ time as described in the proof of Proposition 22.

2-cycle inequalities are separated by enumeration.

Inequalities are separated in the order given above. At a given node of the branch and cut tree, at most 1000 violated inequalities are added to the formulation. There is no limit on the number of violated inequalities of a given class that can be added at a given node.

\subsection{Computational results}

We generated problems with $60, \ldots, 100$ nodes using the AP data set for hub location problems from the OR Library (see [6]). We remove the traffic from a node to itself. We 
Table 1. Improvement due to cuts.

\begin{tabular}{|c|c|c|c|c|c|c|c|c|c|c|c|}
\hline \multicolumn{2}{|c|}{ problem } & \multicolumn{5}{|c|}{$\begin{array}{l}\text { linear knapsack } \\
\text { inequalities }\end{array}$} & \multicolumn{5}{|c|}{$\begin{array}{c}+ \text { cover, quadratic binpacking, triangle } \\
\text { and } \mathrm{W}-2 \text { inequalities }\end{array}$} \\
\hline $\mathrm{c}$ & $\mathrm{n}$ & gap & nod & LP & $\mathrm{CPU}$ & fgap & gap & nod & LP & $\mathrm{CPU}$ & fgap \\
\hline 10 & 59 & 1.16 & 957 & 1552 & 5678 & 0.00 & 0.06 & 3 & 10 & 24 & 0.00 \\
\hline 10 & 69 & 2.43 & 1933 & 2626 & 14400 & 0.57 & 0.40 & 29 & 49 & 363 & 0.00 \\
\hline 10 & 79 & 2.39 & 1033 & 1878 & 14400 & 1.06 & 0.69 & 59 & 128 & 1082 & 0.00 \\
\hline 10 & 89 & 1.91 & 671 & 985 & 14400 & 1.42 & 0.36 & 51 & 140 & 1652 & 0.00 \\
\hline 10 & 98 & 1.81 & 445 & 880 & 14400 & 1.13 & 0.71 & 109 & 311 & 6383 & 0.00 \\
\hline 15 & 59 & 0.85 & 79 & 132 & 296 & 0.00 & 0.39 & 39 & 77 & 246 & 0.00 \\
\hline 15 & 69 & 0.01 & 3 & 5 & 40 & 0.00 & 0.00 & 1 & 3 & 14 & 0.00 \\
\hline 15 & 79 & 0.58 & 341 & 608 & 6897 & 0.00 & 0.37 & 19 & 42 & 350 & 0.00 \\
\hline 15 & 89 & 0.62 & 87 & 154 & 3075 & 0.00 & 0.28 & 17 & 45 & 682 & 0.00 \\
\hline 15 & 99 & 1.09 & 337 & 588 & 13838 & 0.00 & 0.53 & 25 & 72 & 1389 & 0.00 \\
\hline \multicolumn{2}{|c|}{ average } & 1.28 & 588.6 & 940.8 & 8742.4 & 0.42 & 0.38 & 35.2 & 87.7 & 1218.5 & 0.00 \\
\hline
\end{tabular}

Table 2. Results for $c=10$.

\begin{tabular}{lllllllllll}
\hline $\mathrm{n}$ & gap & nod & LP & CPU & con & lk & cov & qbi & tri & w-2 \\
\hline 108 & 0.37 & 25 & 40 & 1939 & 23 & 53 & 33 & 6 & 0 & 4 \\
118 & 0.27 & 29 & 64 & 3240 & 25 & 111 & 86 & 14 & 0 & 1 \\
128 & 0.44 & 25 & 85 & 5047 & 28 & 263 & 147 & 17 & 2 & 7 \\
138 & 0.06 & 19 & 33 & 4239 & 28 & 127 & 80 & 14 & 0 & 7 \\
\hline
\end{tabular}

compute the fixed demands as $a_{i}=\left\lceil\sum_{m \in I} T_{i m}\right\rceil$ for $i \in I$. The capacity of a concentrator is taken to be $M=\left\lfloor\frac{\sum_{i \in I} a_{i}}{n} c\right\rfloor$, where $c \in\{10,15\}$. If there are nodes which have demand more than the capacity of a concentrator, we remove them from the problem.

In Tables $1-4$, we report for each problem:

- $\mathbf{n}$ : the number of nodes with demand less than or equal to $M$

- gap: the duality gap before branching, i.e., $\frac{(u b-d b)}{u b} 100$ where $u b$ is the best upper bound found by the algorithm and $d b$ is the dual bound before branching

- nod: the number of nodes in the branch and cut tree

- LP: the number of LP's solved

- CPU: the CPU time to solve the problem. There is a time limit of 4 hours.

- fgap: the final gap which is $\frac{(u b-l b)}{u b} 100$ where $l b$ is the final lower bound

Initial tests showed that the cover, quadratic binpacking, triangle and $W-2$ inequalities are effective in reducing the solution time. The improvement due to these inequalities is seen in Table 1. We solve the problems, first, with only inequalities (27) and then also with 
Table 3. Results for $c=15$.

\begin{tabular}{lllllllllll}
\hline $\mathrm{n}$ & gap & nod & LP & CPU & con & lk & cov & qbi & tri & w-2 \\
\hline 109 & 0.61 & 51 & 134 & 3537 & 23 & 212 & 102 & 2 & 0 & 0 \\
119 & 0.27 & 5 & 19 & 721 & 24 & 46 & 30 & 11 & 0 & 5 \\
129 & 0.10 & 9 & 27 & 1741 & 26 & 45 & 40 & 4 & 0 & 2 \\
139 & 0.05 & 3 & 14 & 867 & 26 & 33 & 23 & 4 & 0 & 1 \\
149 & 0.07 & 5 & 14 & 1372 & 29 & 32 & 18 & 4 & 0 & 7 \\
158 & 0.00 & 1 & 4 & 431 & 30 & 9 & 6 & 0 & 0 & 0 \\
168 & 0.00 & 1 & 9 & 814 & 31 & 19 & 12 & 1 & 0 & 0 \\
178 & 0.00 & 1 & 6 & 854 & 33 & 13 & 8 & 1 & 0 & 0 \\
188 & 0.01 & 3 & 6 & 2323 & 34 & 12 & 7 & 1 & 0 & 0 \\
\hline
\end{tabular}

Table 4. Results for $c=20$.

\begin{tabular}{lllllllllll}
\hline $\mathrm{n}$ & gap & nod & LP & CPU & con & lk & cov & qbi & tri & w-2 \\
\hline 109 & 0.14 & 17 & 50 & 1223 & 21 & 84 & 47 & 0 & 0 & 4 \\
119 & 0.44 & 21 & 62 & 2848 & 22 & 68 & 39 & 1 & 0 & 13 \\
129 & 0.09 & 7 & 12 & 934 & 23 & 12 & 10 & 0 & 0 & 0 \\
139 & 0.15 & 5 & 10 & 847 & 24 & 16 & 10 & 0 & 0 & 0 \\
149 & 0.00 & 1 & 5 & 390 & 28 & 8 & 7 & 1 & 0 & 2 \\
159 & 0.09 & 7 & 15 & 2367 & 29 & 15 & 14 & 0 & 0 & 0 \\
169 & 0.06 & 15 & 31 & 8366 & 29 & 23 & 21 & 0 & 0 & 0 \\
179 & 0.06 & 7 & 22 & 5405 & 32 & 27 & 26 & 2 & 0 & 4 \\
189 & 0.26 & 5 & 16 & 4920 & 33 & 20 & 14 & 8 & 0 & 0 \\
\hline
\end{tabular}

cover, quadratic binpacking, triangle and $W-2$ inequalities.

As all these problems are solved in less than 2 hours, we generated problems with 110 to 190 nodes. We also test the algorithm on problems with $c=20$. The results are given in Tables 2-4. For each problem, we also report:

- con: number of concentrators installed

- lk: number of violated knapsack inequalities (27)

- cov: number of violated cover inequalities

- qbi: number of violated quadratic binpacking inequalities on three nodes

- tri: number of violated triangle inequalities

- w-2: number of violated $W-2$ inequalities

In Tables 2, 3 and 4, the instances used have the same traffic and cost values. The capacities are different due to different values of $c$. The number of nodes in the problem can also be different as a result of different capacities (since we discard nodes with demand more than the capacity to be feasible). 
In Table 2, we give the results for problems with $c=10$. The problems with 150 and more nodes are not solved to optimality in 4 hours.

The results for problems with $c=15$ and $c=20$ are given in Tables 3 and 4 , respectively. All these problems are solved to optimality in less than 2 hours and 20 minutes. For all types of problems, the linear knapsack and the cover inequalities are the most violated inequalities.

We observe that the difficulty of the problem depends highly on the tightness of capacity constraints. If the capacities are not very tight, the branch and cut algorithm is able to solve problems of 190 nodes in reasonable time. For tight capacities, the algorithm can solve problems of medium sizes.

\section{Appendix}

First, we give a property that helps us to reduce the domain of the separation problem. Let $A_{v}$ be the arc set for the first $v$ triangles.

Proposition 26. If a fractional solution $x$ violates a $k$-triangle inequality, then there exists $a$ violated $k$-triangle inequality where $x_{2 i-1,2 i}>0$ for all $i=1,2, \ldots, k$.

Proof: Assume that fractional solution $x$ violates a k-triangle inequality. If $x_{2 i-1,2 i}>0$ for all $i=1,2, \ldots, k$, then we are done. Otherwise, let $v$ be the smallest index such that $x_{2 v-1,2 v}=0$. We remove the triangles $v, . ., k$ and still have a violated inequality since $0<\sum_{(i, j) \in A_{k}} x_{i j}-k=\sum_{(i, j) \in A_{v-1}} x_{i j}+\sum_{i=v}^{k}\left(x_{2 i, 2 i+1}+\sum_{(2 i+1, j) \in A_{k}} x_{2 i+1, j}\right)-k \leq$ $\sum_{(i, j) \in A_{v-1}} x_{i j}+k-v+1-k=\sum_{(i, j) \in A_{v-1}} x_{i j}-v+1$.

Proof of Theorem 4: The proof is by reduction from the linear ordering problem.

Suppose we are given a set of items $N$ and a set of $\operatorname{arcs} \bar{A}=\{(i, j): i \in N, j \in N \backslash\{i\}\}$. Let $w_{i j} \geq 0$ denote the weight of $\operatorname{arc}(i, j) \in \bar{A}$. The recognition version of the linear ordering problem is as follows: Does there exist a linear ordering $L$ on $N$ such that $\sum_{(i, j) \in O_{L}} w_{i j} \geq B$ (equivalently, $\sum_{(i, j) \in O_{L}} w_{i j}>B-\varepsilon$ for a small enough $\varepsilon>0$ ) where $(i, j) \in O_{L}$ if $i$ comes after $j$ in $L$ ? This problem is NP-complete (see [10] problem [GT8]). Now given an instance of the linear ordering problem, divide all the values $w_{i j}$ and $B$ by a very large number so that $2|N| B<1$. The problem obtained by this scaling is equivalent to the original problem in the sense that the ordering that solves one solves also the other. Now, to avoid the trivial cases, we assume that $B>\sum_{j \in N} w_{i j}$ for each $i \in N$ and that $B>w_{i j}+\sum_{m \in I \backslash\{i, j\}} w_{j m}$ for each arc $(i, j) \in \bar{A}$.

The separation problem for the k-triangle inequalities is as follows: Given $x$ does there exist a violated k-triangle inequality? The number of k-triangle inequalities is exponential in $n$ and given any violated k-triangle inequality, we can verify in polynomial time that it is violated. So the problem is in NP.

Given an instance of the linear ordering problem, for each node $i \in N$, create a dummy node $i^{\prime}$ and set $x_{i i^{\prime}}=1-2 B$. Create also three nodes, $o_{1}, o_{2}, o_{3}$ and set $x_{o_{1} o_{2}}=1-|N| B$, $x_{o_{2} o_{3}}=(|N|-1) B+\varepsilon$ and $x_{o_{1} i}=x_{o_{3} i}=B$ for all $i \in N$. Set also $x_{i j}=w_{i j}$ for all $(i, j) \in \bar{A}$. Define $N^{\prime}=N \cup\left\{i^{\prime}: i \in N\right\} \cup\left\{o_{1}, o_{2}, o_{3}\right\}$ and $A^{\prime}=\left\{(i, j): i \in N^{\prime}, j \in N^{\prime} \backslash\{i\}\right\}$. 


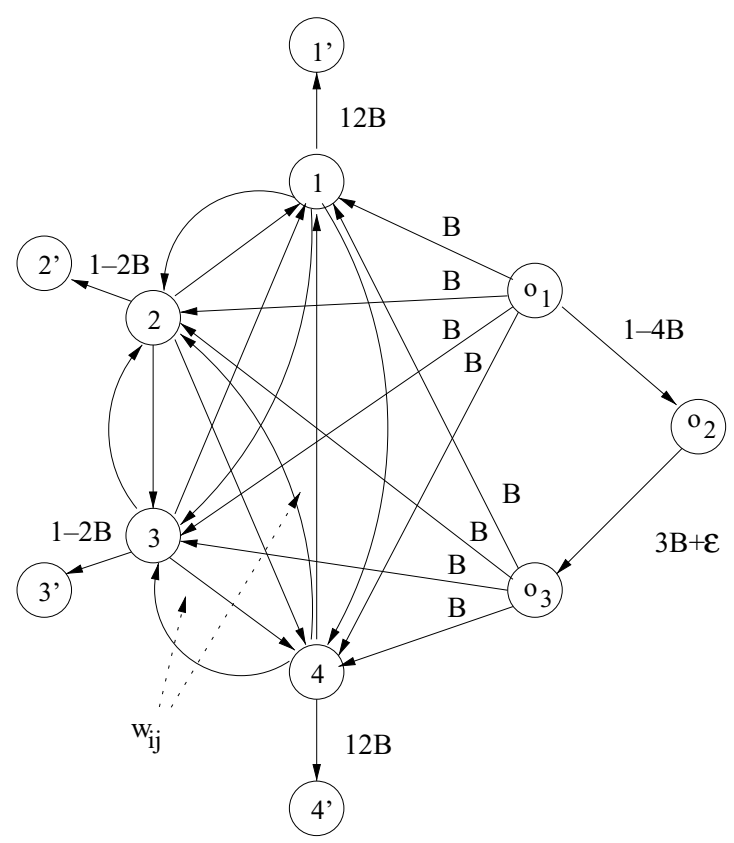

Figure 8. Reducing the linear ordering instance to a fractional solution of UCL

All other $\operatorname{arcs}(i, j) \in A^{\prime}$ have $x_{i j}=0$. In figure 8 , we see the fractional solution of UCL created from an instance of the linear ordering problem on four nodes (the arcs with zero cost are removed).

We claim that there exists a k-triangle inequality violated by $x$ if and only if there exists a linear ordering $L$ on $N$ with $\sum_{(i, j) \in O_{L}} w_{i j}>B-\varepsilon$.

Given a k-triangle configuration with $2 k+1$ nodes, we renumber the nodes from 1 to $2 k+1$. Nodes $2 i-1$ for $i=1, \ldots, k+1$ are odd nodes and the remaining nodes are even nodes. Node $2 i$ is called the even node of node $2 i-1$. Removing nodes $2 i-1$ and $2 i$ from a k-triangle configuration means to remove these nodes and the arcs incident at them and to add arc $(2 i-2,2 i+1)$.

Suppose that we are given a linear ordering $L$ on $N$ with $\sum_{(i, j) \in O_{L}} w_{i j}>B-\varepsilon$. We construct a $(|N|+1)$-triangle configuration. Let the odd nodes be the nodes in $N$ ordered as in $L$ and for each odd node let the even node be its dummy node. Append the triangle $o_{1}, o_{2}, o_{3}$ at the end. Then as $\sum_{(i, j) \in A_{|N|+1}} x_{i j}=|N|(1-2 B)+\sum_{(i, j) \in O_{L}} w_{i j}+1-|N| B+$ $(|N|-1) B+\varepsilon+2|N| B=|N|+\sum_{(i, j) \in O_{L}} w_{i j}+1-B+\varepsilon>|N|+1$, the $(|N|+1)$-triangle inequality is violated.

Now assume that we have a violated k-triangle inequality. By Proposition 26 we can assume that $x_{2 i-1,2 i}>0$ for $i=1,2, \ldots, k$. This implies that a dummy node can be an odd node only if it is the last node since all of its outgoing arcs have value 0 . Assume that the last node is a dummy node. As all its outgoing arcs have value 0 , we can then remove the last triangle and still have a violated inequality. So we look at the case where none of the odd 
nodes is a dummy node. Suppose node $i \in N$ is an odd node. Its even node is either $i^{\prime}$ or some node $j \in N$. Assume that the latter is the case. If the next odd node is $l \in N$, then changing node $j$ to node $i^{\prime}$ cannot decrease the violation since $1-2 B \geq w_{i j}+w_{j l}$ for all $j, l \in N$ as $w_{t s}<B$ for all $t, s \in N$ and $2|N| B<1$. If the next odd node is one of the nodes $o_{1}, o_{2}$ and $o_{3}$, then as $1-2 B \geq w_{i j}$, we can change node $j$ by node $i^{\prime}$ without decreasing the violation.

Now we show that removing nodes $o_{1}, o_{2}$ and $o_{3}$ from where they are and putting them as the last triangle does not decrease the violation. If none of these nodes are used in the k-triangle configuration, then $\sum_{(i, j) \in A_{k}} x_{i j}-k=\sum_{(i, j) \in A_{k} \cap \bar{A}} w_{i j}+k(1-2 B)-k=$ $\sum_{(i, j) \in A_{k} \cap \bar{A}} w_{i j}-2 B k>0$. As all odd nodes are in $N$, we already have a linear ordering on a subset of $N$ with $k+1$ nodes which has cost more than $2 B k$. This is impossible since we assumed that $\sum_{j \in N \backslash\{i\}} w_{i j} \leq B$.

Assume that node $o_{2}$ is the last node. Then the most advantageous case is to have $o_{3}, j, o_{2}$ as the last triangle since $\left(\mathrm{O}_{2}, \mathrm{O}_{3}\right)$ is the only outgoing arc of $\mathrm{O}_{2}$ with nonzero value and all $\operatorname{arcs}\left(o_{3}, j\right)$ with $j \in N$ have value $B$ and the remaining outgoing arcs of $o_{3}$ have value 0 . Even in this case we can remove the last triangle since $B+(|N|-1) B+\varepsilon<1$. So we can assume that $o_{2}$ is not the last node.

Assume that only one of these three nodes $o_{1}, o_{2}$ and $o_{3}$ is used. Then it is either $o_{1}$ or $o_{3}$ since all arcs incident at node $o_{2}$ have value 0 except $\left(o_{1}, o_{2}\right)$ and $\left(o_{2}, o_{3}\right)$, so it can only be the last node and can be removed in this case as explained above. The node which is $o_{1}$ or $o_{3}$ must be an odd node, with the even node being a node $j \in N$. As $|N| B+w_{j t}<1$ (since $w_{j t}<B$ and $2|N| B<1$ ) for all $t \in N$ removing this node and its even node cannot decrease the violation.

Assume now two of these nodes are used. If these two nodes are $o_{1}$ and $o_{2}$, then $o_{1}$ is an odd node and $o_{2}$ is its even node. In this case we can remove these two nodes since at most we can have $1-|N| B+|N| B=1$ on the triangle. If the nodes are $o_{2}$ and $o_{3}$, then $o_{2}$ has to be the odd node and $o_{3}$ has to be its even node. In this case $(|N|-1) B+\varepsilon+B \leq 1$. So we can again remove these two nodes. If we have nodes $o_{1}$ and $o_{3}$, then as the arcs connecting them have value 0 , we should have them both as odd nodes. As this is the same as having them separately, they can be removed.

If all three nodes are used and if they are not together, then node $o_{2}$ has to be used with $o_{1}$ or $o_{3}$. So we are in one of the cases above. If these nodes are used together, it is either the triangle $o_{1}, o_{2}, o_{3}$ or the triangle $o_{2}, o_{3}, o_{1}$ since $x_{o_{1} o_{3}}=0, x_{o_{3} o_{1}}=0, x_{o_{3} o_{2}}=0$ and $x_{o_{2} o_{1}}=0$. In the latter case, changing the triangle to $o_{1}, o_{2}, o_{3}$ does not decrease the violation. Finally moving this triangle to the end of the sequence cannot decrease the violation. If these nodes are removed from the $\mathrm{k}$-triangle configuration, then we append this triangle at the end since it has value $(|N|-1) B+\varepsilon+1-|N| B+2 k B=\varepsilon+1-B+2 k B>1$ when $k \geq 1$.

This implies that we obtain a k-triangle configuration where the odd nodes except $o_{1}$ and $o_{3}$ are in $N$. If we have $k$ triangles, we get a linear ordering on $k-1$ nodes that we call $L_{k-1}$. Then $\sum_{(i, j) \in A_{k}} x_{i j}-k=(k-1)(1-2 B)+\sum_{(i, j) \in O_{L_{k-1}}} w_{i j}+1-|N| B+(|N|-$ $1) B+\varepsilon+2(k-1) B-k=\sum_{(i, j) \in O_{L_{k-1}}} w_{i j}-B+\varepsilon>0$. This partial linear ordering can be completed by adding the remaining nodes to the end of the order and it gives a linear ordering $L$ with $\sum_{(i, j) \in O_{L}} w_{i j}>B-\varepsilon$. 


\section{Acknowledgments}

This research was partially supported by France Telecom R \& D under contract no. 99 1B 774. Their support is gratefully acknowledged.

\section{References}

1. K. Aardal, Y. Pochet, and L.A. Wolsey, "Capacitated facility location: Valid inequalities and facets," Mathematics of Operations Research, vol. 20, pp. 562-582, 1995.

2. K. Aardal, "Capacitated facility location: Separation algorithms and computational experience," Mathematical Programming, vol. 81, pp. 149-175, 1998.

3. P. Avella and A. Sassano, "On the p-median polytope," Mathematical Programming, vol. 89, pp. 395-411, 2001.

4. E. Balas, "Facets of the Knapsack Polytope," Mathematical Programming, vol. 8, pp. 146-164, 1975.

5. E. Balas and J.B. Mazzola, "Nonlinear 0-1 Programming: I. Linearization Techniques," Mathematical Programming, vol. 30, pp. 1-21, 1984.

6. J.E. Beasley, "OR-Library: distributing test problems by electronic mail," Journal of the Operational Research Society, vol. 41, pp. 1069-1072, 1990.

7. G. Carello, F. Della Croce, M. Ghirardi, and R. Tadei, "Solving hub location problem in telecommunication network design: A local search approach,” Networks, vol. 44, pp. 94-105, 2004.

8. Th. Christof, Ein Verfharen zur Transformation Zwischen Polyederdarstellungen, Diplomarbeit, Universität Augsburg, 1991.

9. Q. Deng and D. Simchi-Levi, "Valid inequalities, facets and computational results for the capacitated concentrator location problem," Department of Industrial Engineering and Operations Research, Columbia University, New York, NY 10027-6699, 1992.

10. M.R. Garey and D.S. Johnson, Computers and Intractability: A Guide to the Theory of NP-Completeness, Freeman, New York, 1979.

11. E. Gourdin, M. Labbé, and H. Yaman, Telecommunication and Location. In Facility Location: Applications and Theory, Z.Drezner and H.W. Hamacher (Eds.), Springer, pp. 275-305, 2002.

12. Z. Gu, G.L. Nemhauser and M.W.P. Savelsbergh, "Cover Inequalities for 0-1 Linear Programs: Computation," INFORMS Journal on Computing, vol. 10, pp. 427-437, 1998.

13. P.L. Hammer, E.L. Johnson, and U.N. Peled, "Facets of Regular 0-1 Polytopes," Mathematical Programming, vol. 8, pp. 179-206, 1975.

14. M. Júnger and S. Thienel, "The ABACUS system for branch-and-cut-and-price algorithms in integer programming and combinatorial optimization," Softw. Pract. Experience, vol. 30, pp. 1325-1352, 2000.

15. M. Labbé, H. Yaman, and E. Gourdin, "A branch and cut algorithm for hub location problems with single assignment," Mathematical Programming, vol. 102, pp. 371-405, 2005.

16. J.M.Y. Leung and T.L. Magnanti, "Valid inequalities and facets of the capacitated plant location problem," Mathematical Programming, vol. 44, pp. 271-291, 1989.

17. S. Martello and P. Toth, Knapsack Problems: Algorithms and Computer Implementations, Wiley, New York, 1990.

18. G.L. Nemhauser and L.A. Wolsey, Integer and Combinatorial Optimization, Wiley, New York, 1988.

19. M.W. Padberg, "On the facial structure of set packing polyhedra," Mathematical Programming, vol. 5, pp. 199-215, 1973.

20. L. Wolsey, "Faces for a linear inequality in 0-1 variables," Mathematical Programming, vol. 8, pp. 165-178, 1975.

21. H. Yaman, Concentrator Location in Telecommunications Networks, Springer, 2005. 